\title{
Key Parameters Estimation and Adaptive Warning Strategy for Rear-End Collision of Vehicle
}

\author{
Xiang Song, Xu Li, and Weigong Zhang \\ School of Instrument Science and Engineering, Southeast University, Nanjing 210096, China \\ Correspondence should be addressed to Xu Li; lixu.mail@163.com
}

Received 26 May 2015; Revised 31 August 2015; Accepted 20 September 2015

Academic Editor: Juan C. Agüero

Copyright (C) 2015 Xiang Song et al. This is an open access article distributed under the Creative Commons Attribution License, which permits unrestricted use, distribution, and reproduction in any medium, provided the original work is properly cited.

\begin{abstract}
The rear-end collision warning system requires reliable warning decision mechanism to adapt the actual driving situation. To overcome the shortcomings of existing warning methods, an adaptive strategy is proposed to address the practical aspects of the collision warning problem. The proposed strategy is based on the parameter-adaptive and variable-threshold approaches. First, several key parameter estimation algorithms are developed to provide more accurate and reliable information for subsequent warning method. They include a two-stage algorithm which contains a Kalman filter and a Luenberger observer for relative acceleration estimation, a Bayesian theory-based algorithm of estimating the road friction coefficient, and an artificial neural network for estimating the driver's reaction time. Further, the variable-threshold warning method is designed to achieve the global warning decision. In the method, the safety distance is employed to judge the dangerous state. The calculation method of the safety distance in this paper can be adaptively adjusted according to the different driving conditions of the leading vehicle. Due to the real-time estimation of the key parameters and the adaptive calculation of the warning threshold, the strategy can adapt to various road and driving conditions. Finally, the proposed strategy is evaluated through simulation and field tests. The experimental results validate the feasibility and effectiveness of the proposed strategy.
\end{abstract}

\section{Introduction}

Vehicle and road safety has been a key issue for the communities and governments. With emerging new technologies and knowledge, the advanced driver assistance systems (ADAS) $[1,2]$ have been proposed to reduce road accidents and improve vehicle safety. Since the rear-end collisions account for nearly $35 \%$ of all traffic accidents, the frontal collision warning/avoidance (FCW/FCA) systems can be considered as one of the main research orientations in ADAS nowadays [3].

The FCW/FCA systems are designed to give a warning and/or perform autonomous braking when a collision is imminent [4]. In the literature, there are two possible solutions from different levels, that is, the sensor level and the warning algorithm level [5]. In other words, a well-designed FCW/FCA system collects environmental information by the in-vehicle sensors and makes quick and reasonable suggestions through the warning algorithm. To this end, there has been great interest in the environment perception technology and the warning algorithm. Compared with the related sufficient existing studies $[6,7]$ of the environment perception technology, the warning algorithm is still an open issue to be addressed [8]. The main reason is that the FCW/FCA systems are being used in a wide range of different areas and under very different circumstances; thus the existing algorithms do hardly meet the practical requirements in various driving environments $[9,10]$.

Since the early 1990s, many FCW/FCA algorithms have been proposed [11]. Generally speaking, these algorithms can be divided into two major types, that is, distance-based algorithm and time-based algorithm [12]. At the current speed of the vehicle, the distance can be converted into the time domain; therefore, only the distance-based algorithm is discussed in this paper, and the proposed methodology can be easily extended to the time-based algorithm. The distance-based warning algorithm generally employs a twostep approach, namely, the calculation of the warning threshold or the safety distance and then the comparison between the warning threshold and the actual distance. Since the 
actual distance from the leading vehicle (LV) to the subject vehicle (SV) can be measured by the distance sensors such as radar, how to determine the safety distance is the main challenge of the distance-based warning algorithm.

In the existing FCW/FCA systems, the typical distancebased warning algorithms contain the fixed distance algorithm, the fixed time-headway algorithm [13], the kinematicsbased algorithm [14], the numerical algorithm [15], and the driver model algorithm [16]. In addition, some artificial intelligence- (AI-) based algorithms have been developed to calculate the warning threshold in recent years, such as artificial neural network-based algorithm [17] and expert systembased algorithm [18]. However, these AI-based algorithms often require expert experience or prior knowledge, which may limit their practical application.

Both the fixed distance algorithm and the fixed timeheadway algorithm can adapt to the condition that the relative speed between SV and LV is low. However, the calculated safety distance is small and may lead to false negative (missed warning) in the condition of high relative speed. The numerical and driver algorithms model the nonlinear relationship between the warning threshold and the measured values, that is, speed, acceleration, and so forth, by fitting curves. Obviously these algorithms require a lot of experience or training data, so they are difficult to adapt to complex traffic environment and different drivers.

Among the existing distance-based algorithms, the kinematics-based algorithms are most widely used in the actual application. The kinematics-based algorithms calculate the warning threshold by analyzing the relative motion between SV and LV. The most well-known algorithms, such as Mazda algorithm, Honda algorithm, and Berkeley improved algorithm, belong to this category. However, each algorithm discussed above has its particular suitable condition and may cause large errors in other conditions. For example, Mazda algorithm is developed to suit the emergency stop condition of LV; thus the calculated safety distance in other conditions is too large and the warning time is too early, which may lead to false positive (false warning). Meanwhile, the calculated safety distances of the Honda algorithm and the Berkeley improved algorithm are too small and may lead to false negative.

It can be seen that there are some deficiencies in the existing algorithms discussed above. The main reason is that each algorithm is only suitable for a certain condition and unable to adapt to other conditions [19]. In others words, these algorithms do not have the adaptability to accommodate the change of the driving condition. To the author's knowledge, it can be attributed to the fact that it is difficult to accurately obtain some key parameters under the existing technical conditions. These parameters are often treated as constant and may deviate from the true value in some driving conditions, which may influence the reliability of the existing algorithms.

(1) These algorithms are difficult to adapt to the change of the road friction coefficient. The road friction coefficient is directly related to the maximum deceleration of SV and then affects the warning threshold. Although Tang and Yip [20] have proposed that the maximum deceleration should be adjusted according to different road friction coefficients, there is no effective way to measure the road friction coefficient for the FCW/FCA systems.

(2) The acceleration of LV or the relative acceleration between SV and LV cannot be accurately obtained; hence the algorithms are difficult to adapt to various driving states of LV. For example, in the acceleration and deceleration cases of $\mathrm{LV}$, the corresponding calculation method for safety distance should be different.

(3) Since the driver's reaction time is usually considered as constant value, these algorithms are difficult to distinguish different drivers and driver's states.

As these key parameters are time-varying during vehicle operation and unable to be measured directly, the warning threshold of existing algorithms cannot be adaptively adjusted according to the actual driving conditions, which may result in serious false warning in complex traffic environment.

To realize reliable rear-end collision warning for the vehicle in complex traffic environments, this paper proposes an adaptive strategy based on the kinematics analysis of LV and SV. This strategy aims to address the practical aspects of the collision warning problem. In this strategy, both algorithms for key parameters estimation and the warning decision are developed to obtain higher performance. The novel aspects of this paper can be summarized as follows.

(1) Several key parameters estimation algorithms are developed to provide more accurate and richer information for subsequent warning method. They include a two-stage algorithm which contains a Kalman filter and a Luenberger observer for relative acceleration estimation, a Bayesian theory-based algorithm of calculating the road friction coefficient, and an artificial neural network for the determination of the driver's reaction time. Due to the parameters estimation algorithms in this paper, the information can be obtained more accurately in a wide range of driving circumstances, and then the performance and adaptability of subsequent warning method can be significantly improved.

(2) A variable-threshold method is proposed to realize reliable rear-end collision warning. In the implementation of variable-threshold method, the motion of LV is analyzed, and the warning threshold is calculated according to the driving state of LV, that is, acceleration, deceleration, and uniform motion. Compared with the existing kinematics-based methods, this method can adapt to different driving conditions due to the accurate estimation of the crucial parameters. Meanwhile, the warning threshold can be adjusted in accordance with the driving state of $\mathrm{LV}$, which can remarkably reduce the missed warning and false warning.

It should be noted that this paper only focuses on the warning algorithm level of rear-end collision warning systems. However, the proposed strategy is not limited to the sensors described in this paper. Other advanced technologies, such as vehicle to vehicle communication $(\mathrm{V} 2 \mathrm{~V})$, can also be applied to the proposed strategy.

The remainder of the paper is organized as follows. Section 2 gives an overview of the proposed strategy. The estimation algorithms of crucial parameters are discussed in detail in Section 3. Section 4 presents the variable-threshold 


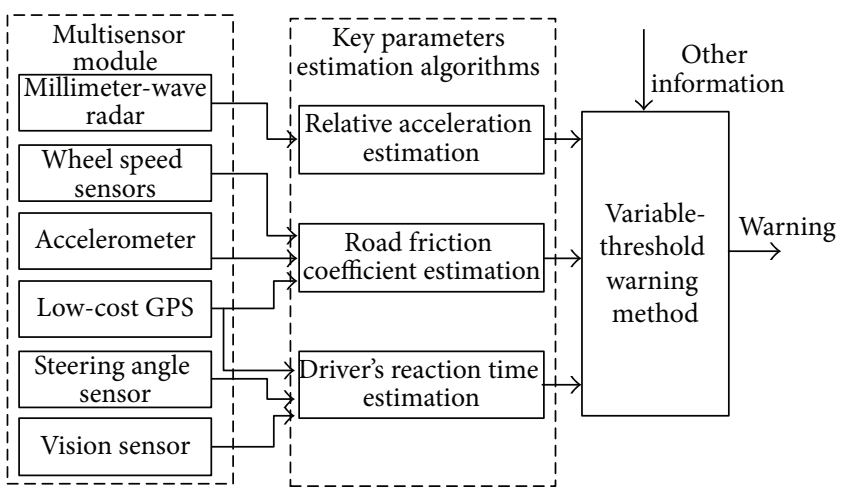

FIgURE 1: Proposed strategy for rear-end collision warning.

method. Simulation and experimental results are provided in Sections 5 and 6 . Section 7 is devoted to the conclusion.

\section{Proposed Warning Strategy}

The proposed strategy is shown in Figure 1. Figure 1 is mainly composed of three parts, that is, multisensor module, key parameters estimation algorithms, and variable-threshold method for warning decision.

The multisensor module includes such sensors as millimeter-wave radar, wheel speed sensors, and low-cost GPS. The millimeter-wave radar provides the distance and relative speed between SV and $\mathrm{LV}$ with $20 \mathrm{~Hz}$ update rate. The low-cost GPS provides speed information of SV, and the vision sensor only provides the path offset information of SV. The accelerometer is only along the longitudinal axes in vehicle body frame.

Several estimation algorithms are developed to acquire the information of key parameters. First, a two-stage algorithm is designed to obtain accurate relative acceleration between SV and LV; thus the acceleration of LV can be calculated. The two-stage algorithm contains a Kalman filter and a Luenberger observer. Both the Kalman filter and Luenberger observer have been verified to be effective and robust for the system. Then, a Bayesian theory-based algorithm is presented to estimate the road friction coefficient which can represent the different types of road. The road friction coefficient directly affects the maximum braking deceleration and thus affects the calculation of the safety distance. In addition, a Back Propagation (BP) artificial neural network is designed to determine the driver's reaction time. These parameters' estimation algorithms can provide richer information for the subsequent warning method.

The variable-threshold method is proposed to realize the warning decision. Through the kinematics analysis of the relative motion between $\mathrm{LV}$ and $\mathrm{SV}$, the warning threshold or safety distance is calculated in real-time. Due to the previous estimation of crucial parameters, the warning distance can be adaptively adjusted according to various road conditions and different driver's states. Further, the variable-threshold method, respectively, calculates the safety distance according to the different driving states of SV, that is, acceleration, deceleration, and uniform motion, so it can remarkably reduce the missed warning and false warning compared with existing methods.

\section{Key Parameters Estimation Algorithms}

As discussed above, there are three key parameters that may affect the performance of the warning strategy, that is, the relative acceleration, the road friction coefficient, and the driver's reaction time.

3.1. Estimation of Relative Acceleration. Accurate identification of LV's driving state (i.e., accelerated motion state, constant speed state, and decelerated motion state) can improve the adaptability of the subsequent warning method. The calculation method of the safety distance should be different under the different states of LV. Meanwhile, the accurate estimation of the value of LV's acceleration can improve the accuracy of the safety distance. The acceleration of LV can be calculated by the relative acceleration plus SV'S acceleration. However, the relative acceleration is hardly to be measured directly under the existing technologies. In the related literature, the relative acceleration is usually obtained by the following methods.

(1) The relative acceleration is calculated by the direct differentiation of the relative speed. However, this method may cause serious calculation error, data loss, and latency [21].

(2) The model of the relative movement is created, and the filtering methods are used to estimate the relative acceleration. This method is widely used in the field of vehicle tracking, but the effectiveness of this method depends on the accuracy of the model and the validity of the observational information. The commonly used models, such as the constant velocity (CV) model and the constant acceleration (CA) model, cannot accurately describe the change of the relative acceleration. Hence, the estimation error is large [22, 23].

(3) With the development of vehicle-to-vehicle communication (V2V) technology, the acceleration of LV can be transmitted from the LV itself by using the V2V. However, the $\mathrm{V} 2 \mathrm{~V}$ technology is still in the research stage and needs costly additional hardware [24].

A two-stage algorithm is developed to estimate the relative acceleration between SV and LV, as shown in Figure 2. In this algorithm, the relative distance and the relative speed which are measured by radar are used as observational variables, and the relative motion model which can accurately describe the change of the relative acceleration is established. The Kalman filter (KF) is designed to preliminary estimate the relative acceleration utilizing the relative motion model, and then the Luenberger observer is adopted to estimate the preliminary estimation bias based on the relative speed. Therefore, the preliminary estimation result is corrected, and the relative acceleration can be accurately obtained.

Describing the statistical distribution of the relative acceleration is a difficult problem. The commonly used CV and CA models are difficult to describe the dynamic change of the relative acceleration. Therefore, the "current" statistical 


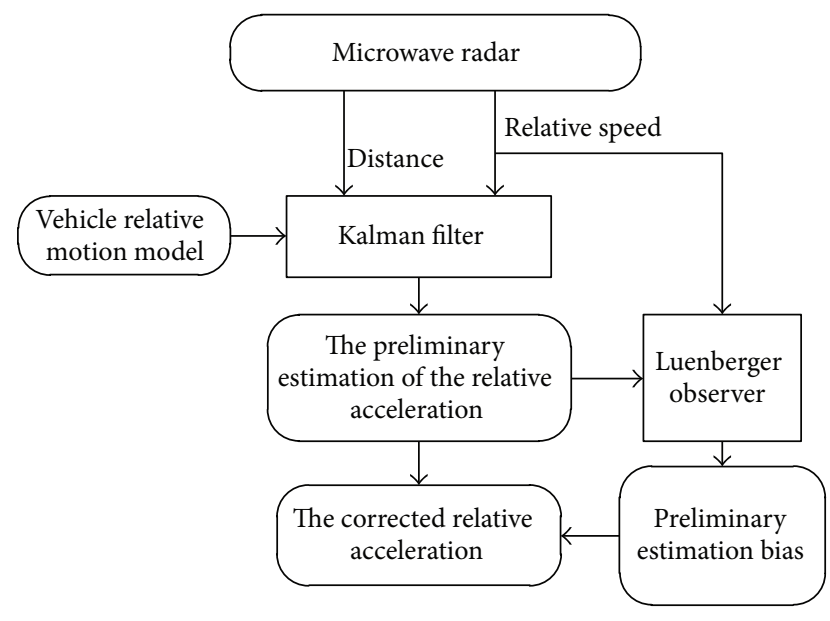

FIGURE 2: The algorithm for relative acceleration estimation.

model which is widely used in the navigation field [25] is adopted in this paper. In this model, the region of the relative acceleration during the time period is limited and around the "current" relative acceleration. The current probability density of the relative acceleration change has the form of modified Raleigh density [26]. The discretized state equation and measurement equation can be written as

$$
\begin{aligned}
& \mathbf{X}(k)=\mathbf{A}(k, k-1) \mathbf{X}(k-1)+\mathbf{U}(k-1)+\mathbf{W}(k-1), \\
& \mathbf{Z}(k)=\mathbf{H}(k) \mathbf{X}(k)+\mathbf{V}(k),
\end{aligned}
$$

where $k$ refers to the discrete-time step; the state vector is $\mathbf{X}=\left[\begin{array}{lll}d & v_{r} & a_{r}\end{array}\right]^{\prime}$, where $d, v_{r}$, and $a_{r}$ are the relative distance, speed, and acceleration between SV and LV, and the measurement vector is $\mathbf{Z}=\left[\begin{array}{ll}d_{\text {radar }} & v_{r_{-} \text {radar }}\end{array}\right]^{\prime}$, where $d_{\text {radar }}$ and $v_{r \text { radar }}$ are the observed relative distance and speed from radar. The state transition matrix $\mathbf{A}[25,27]$, the system input matrix $\mathbf{U}$, and the measurement matrix $\mathbf{H}$ are

$$
\begin{aligned}
\mathbf{A}(k, k-1) & =\left[\begin{array}{ccc}
1 & T & \left(\frac{T}{\tau}-1+e^{-T / \tau}\right) \tau^{2} \\
0 & 1 & \left(1-e^{-T / \tau}\right) \tau \\
0 & 0 & e^{-T / \tau}
\end{array}\right], \\
\mathbf{U}(k-1) & =\left[\begin{array}{c}
0 \\
0 \\
T \cdot \frac{\bar{a}_{r}(k-1)}{\tau}
\end{array}\right], \\
\mathbf{H}(k) & =\left[\begin{array}{lll}
1 & 1 & 0
\end{array}\right]^{\prime},
\end{aligned}
$$

where $T$ is the sampling time period and $\tau$ is the maneuvering time constant. For vehicle movements [27], maneuvering time constant generally ranges from 1 to $20 \mathrm{~s} . \bar{a}_{r}$ is the mean value of the current relative acceleration, and it can be expressed approximately as $\widehat{a}_{r}(k-1)$, which refers to the filter calculation result of $a_{r}(k-1) ; \mathbf{W}$ and $\mathbf{V}$ are the system and measurement noise vectors. The covariance matrix of $\mathbf{W}$ is $\mathbf{Q}(k)=\left(2 \sigma^{2} / \tau\right)\left[\begin{array}{lll}q_{11} & q_{12} & q_{13} \\ q_{11} & q_{22} \\ q_{13} & q_{22} \\ q_{23} & q_{33}\end{array}\right]$. The values of $q_{11}, q_{12}$, $q_{13}, q_{23}$, and $q_{33}$ can be referred to in $[25,26]$. In the actual implementation of the model for vehicle, $\mathbf{Q}(k)$ can be simplified as $\mathbf{Q}(k) \approx\left(2 \sigma^{2} / \tau\right)\left[\begin{array}{ccc}T^{5} / 20 & T^{4} / 8 & T^{3} / 6 \\ T^{4} / 8 & T^{3} / 3 & T^{2} / 2 \\ T^{3} / 6 & T^{2} / 2 & T\end{array}\right]$. According to the modified Rayleigh distributions [27], the square error of relative acceleration can be deduced as

$$
\sigma^{2}= \begin{cases}\left(\frac{4}{\pi}-1\right)\left[a_{\max }-\widehat{a}_{r}(k)\right]^{2} & \widehat{a}_{r}(k)>0 \\ \left(\frac{4}{\pi}-1\right)\left[a_{-\max }+\widehat{a}_{r}(k)\right]^{2} & \widehat{a}_{r}(k)<0 .\end{cases}
$$

$\widehat{a}_{r}(k)$ is the filter calculation result of $a_{r}(k)$ and $a_{\max }$ and $a_{-\max }$ are the maximal positive acceleration and the maximal negative acceleration, respectively; the covariance matrix of $\mathbf{V}$ is $\mathbf{R}(k)=\left[\begin{array}{cc}\sigma_{d}{ }^{2} & 0 \\ 0 & \sigma_{v_{r}}{ }^{2}\end{array}\right]$, where $\sigma_{d}{ }^{2}$ and ${\sigma_{v_{r}}}^{2}$ can be determined according to the measurement noise statistics of radar.

The Kalman filtering process consists of the following two phases:

Time update:

$$
\begin{aligned}
\widehat{\mathbf{X}}(k, k-1)= & \mathbf{A}(k, k-1) \widehat{\mathbf{X}}(k-1)+\mathbf{U}(k-1), \\
\mathbf{P}(k, k-1)= & \mathbf{A}(k, k-1) \mathbf{P}(k-1) \mathbf{A}^{\prime}(k, k-1) \\
& +\mathbf{Q}(k-1)
\end{aligned}
$$

Measurement update:

$$
\begin{aligned}
\mathbf{K}(k)= & \mathbf{P}(k, k-1) \cdot \mathbf{H}^{\prime}(k) \\
& \cdot\left[\mathbf{H}(k) \mathbf{P}(k, k-1) \mathbf{H}^{\prime}(k)+\mathbf{R}(k)\right]^{-1}, \\
\widehat{\mathbf{X}}(k)= & \widehat{\mathbf{X}}(k, k-1) \\
& +\mathbf{K}(k)[\mathbf{Z}(k)-\mathbf{H}(k) \cdot \widehat{\mathbf{X}}(k, k-1)], \\
\mathbf{P}(k)= & {[\mathbf{I}-\mathbf{K}(k) \cdot \mathbf{H}(k)] \cdot \mathbf{P}(k, k-1) . }
\end{aligned}
$$

The relative acceleration, that is, $a_{r_{-} \text {estimate }}$, can be preliminary estimated by using Kalman filter. However, there is large bias in the preliminary estimation result. To calculate the relative acceleration accurately, the bias needs to be estimated and removed from the preliminary estimation result. Note that the relative speed can be accurately obtained from radar. On the other hand, the relative speed can also be obtained by integrating the preliminary estimated relative acceleration $a_{r_{-} \text {sstimate }}$. Due to the bias present in the preliminary estimation signal, the relative speed calculated by the integration of $a_{r-\text { sstimate }}$ usually drifts. A combination of these two signals provides a way to determine the preliminary estimation bias.

A Luenberger observer is designed to estimate the bias. Assume that $a_{r_{-} \text {offset }}$ denotes the estimation bias and that it has a slowly varying characteristic, the state-space equations can be described as follows:

$$
\begin{aligned}
\dot{v}_{r} & =a_{r_{-} \text {estimate }}-a_{r_{-} \text {offset }}, \\
\dot{a}_{r_{-} \text {offset }} & =0 .
\end{aligned}
$$




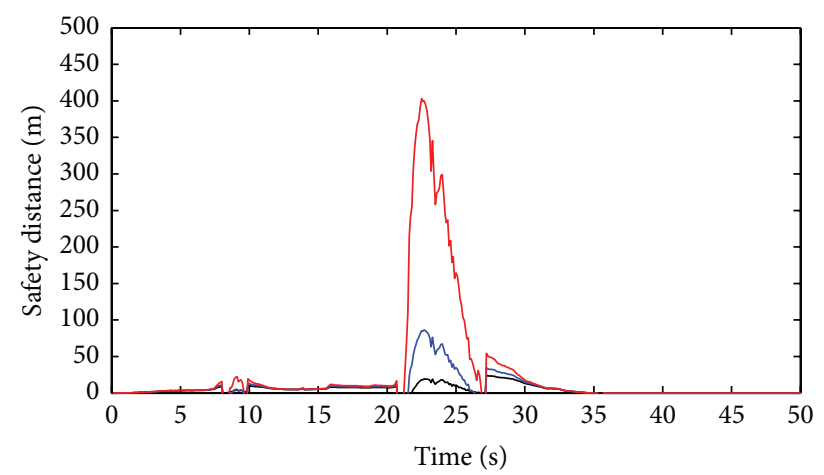

When road friction is 0.8
When road friction is 0.4
When road friction is 0.2

FIGURE 3: The calculation results of the safety distances under different road friction conditions.

Based on (6), the Luenberger observer can be described as

$$
\begin{aligned}
\dot{\hat{v}}_{r} & =a_{r_{-} \text {estimate }}-\widehat{a}_{r_{-} \text {offset }}+L_{1}\left(v_{r_{\text {_radar }}}-\widehat{v}_{r}\right), \\
\dot{\hat{a}}_{r_{-} \text {offset }} & =L_{2}\left(v_{r_{-} \text {radar }}-\widehat{v}_{r}\right),
\end{aligned}
$$

where $\widehat{v}_{r}$ and $\widehat{a}_{r_{-} \text {offset }}$ are the estimated values of the relative speed and the relative acceleration bias and $L_{1}$ and $L_{2}$ are the observer feedback gains. The relative acceleration bias, $a_{r \text { offset }}$, can be estimated and used to correct the preliminary estimated relative acceleration; that is, the final estimation result of the relative acceleration is $a_{r}=a_{r_{\text {_estimate }}}-a_{r_{\text {-offset }}}$.

\subsection{Bayesian Theory-Based Road Friction Coefficient Deter-} mination. The longitudinal road friction coefficient is an essential parameter for the vehicle braking performance. It directly affects the maximum braking deceleration and thus affects the safety distance. For example, if other conditions are the same, the safety distance in low friction coefficient road is obviously larger than that in high friction coefficient road. To illustrate the effect of the road friction coefficient on the calculation of the safety distance. One test under different road friction coefficients is shown in Figure 3.

It can be seen in Figure 3 that if other conditions are the same, the safety distance is very different with the change of road friction coefficient, that is, up to hundreds of meters, which can remarkably influence the performance of the warning algorithm. Hence, to meet the adaptability requirement of FCW/FCA systems, the road friction coefficient must be accurately estimated under normal driving conditions in real-time.

Although Tang and Yip [20] have pointed out that the maximum deceleration should be adjusted according to different road conditions, there is no effective way to accurately obtain the road friction coefficient for FCW/FCA systems.

In the field of active safety control systems such as antilock braking system (ABS), the road friction coefficient estimation method based on the vehicle longitudinal

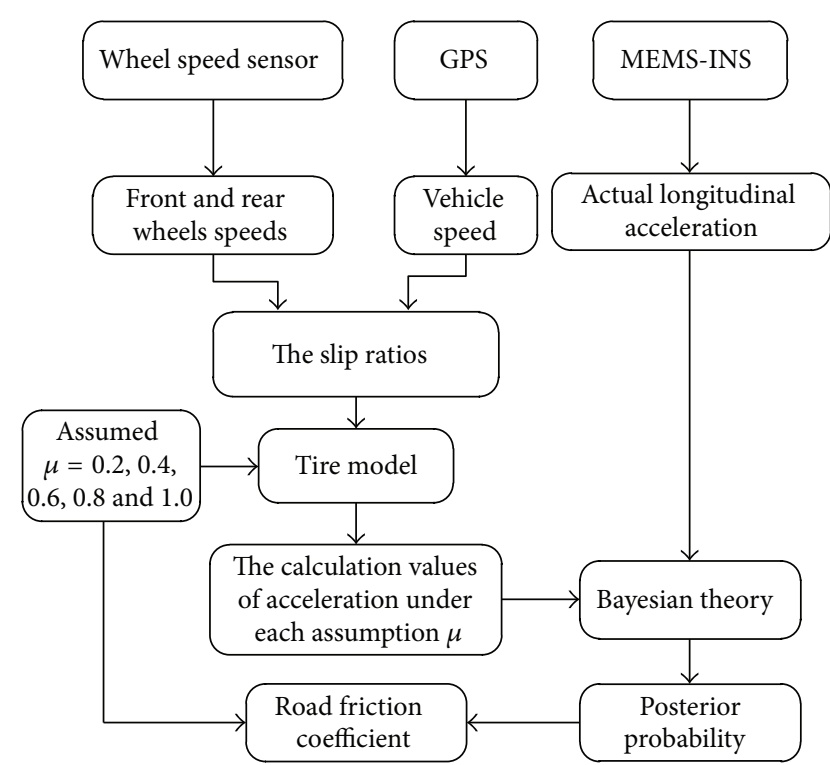

FIGURE 4: Flowchart of the road friction coefficient estimation.

dynamics is most feasible $[28,29]$. The relationship between normalized longitudinal tire force and slip ratio is different under different road conditions, which is the basis of utilizing the vehicle longitudinal dynamics to estimate road friction coefficient [28].

The most well-known research in this area is on the use of "slip-slope" for friction coefficient identification [28]. However, this method is only suitable for the condition of the low slip ratios. The parameter estimation method is another commonly used method [29]. But only at the large slip ratios, the estimation results will be approximate to the true value. Therefore, the main problem of the road friction coefficient estimation algorithms is that these algorithms cannot be applied to both high and low slip ratios simultaneously. Meanwhile, these algorithms are not suitable for rear-end collision warning system due to the use of expensive sensors [29] or large computational complexity [30].

To solve these problems, an estimation algorithm based on the Bayesian theory is proposed in this paper. This algorithm utilizes the relationship between normalized longitudinal tire force and slip ratio to identify the longitudinal tireroad friction coefficient, which can quickly and accurately estimate the longitudinal road friction coefficient both at high and low slip ratio conditions, as shown in Figure 4.

Assuming that all wheels are under the same road surface condition, it means that the friction coefficient is assumed to be the same at each wheel of the SV, which is true for many driving situations.

If only longitudinal motion is considered and the lateral force is ignored, the normalized longitudinal tire force $s$ and slip ratio $\varphi$ at each wheel can be represented as [31]

$$
\begin{aligned}
& s=\frac{\left(\omega r-v_{x}\right)}{\max \left(\omega r, v_{x}\right)}, \\
& \varphi=\frac{F_{x}}{F_{z}},
\end{aligned}
$$


where $\omega$ is the angular wheel speed; $r$ is the tire radius; $v_{x}$ is the speed of SV; $F_{x}$ is the longitudinal force from ground to wheel; and $F_{z}$ is the normal force.

The proposed estimation algorithm for road friction coefficient is described as follows:

(1) The longitudinal slip ratios of front and rear wheel axles, $s_{f}$ and $s_{r}$, are calculated utilizing (8), respectively.

(2) A simplified magic formula tire model $[32,33]$ is adopted and the model can be described as

$$
\varphi=\mu \sin [C \arctan (B s)],
$$

where $\mu$ is the road friction coefficient and $B$ and $C$ are the model parameters.

Therefore, the normalized longitudinal tire forces of the front and rear wheel axles can be described as

$$
\begin{aligned}
\varphi_{f} & =\frac{F_{x f}}{F_{z f}}=\mu \sin \left[C \arctan \left(B s_{f}\right)\right], \\
\varphi_{r} & =\frac{F_{x r}}{F_{z r}}=\mu \sin \left[C \arctan \left(B s_{r}\right)\right],
\end{aligned}
$$

where $F_{z f}$ and $F_{z r}$ are the normal forces at front and rear tires; they can be calculated as follows:

$$
\begin{aligned}
& F_{z f}=\frac{m g l_{2}}{\left(l_{1}+l_{2}\right)}, \\
& F_{z r}=\frac{m g l_{1}}{\left(l_{1}+l_{2}\right)},
\end{aligned}
$$

where $l_{1}$ and $l_{2}$ are the distances from the center of gravity to the front and the rear axles.

Due to the assumption that the front and rear tires are on the same road condition, the total longitudinal force is

$$
\begin{aligned}
F_{x} & =F_{x f}+F_{x r}=\varphi_{f} F_{z f}+\varphi_{r} F_{z r} \\
& =\mu\left\{F_{z f} \sin \left[C \arctan \left(B s_{f}\right)\right]\right. \\
& \left.+F_{z r} \sin \left[C \arctan \left(B s_{r}\right)\right]\right\} .
\end{aligned}
$$

Assume that the road friction coefficients are $\mu_{1}=$ $0.2, \mu_{2}=0.4, \mu_{3}=0.6, \mu_{4}=0.8$, and $\mu_{5}=1.0$, respectively. For the five assumed road friction coefficients, the corresponding assumed simplified magic formula [32] tire models are established, as shown in Figure 5. In the $i$ th ( $i=1,2,3,4$, and 5) assumed model, the longitudinal force can be calculated as

$$
\begin{aligned}
F_{x i} & =\mu_{i}\left\{F_{z f} \sin \left[C \arctan \left(B s_{f}\right)\right]\right. \\
& \left.+F_{z r} \sin \left[C \arctan \left(B s_{r}\right)\right]\right\} \quad(i=1,2,3,4,5) .
\end{aligned}
$$

The longitudinal vehicle dynamics model [34] can be written as

$$
m a_{x}=F_{x}-D_{a} v^{2}-C_{\text {roll }} m g,
$$

where $m$ is the mass of the vehicle; $a_{x}$ is the real-time vehicle longitudinal acceleration; $D_{a}$ is the air resistance

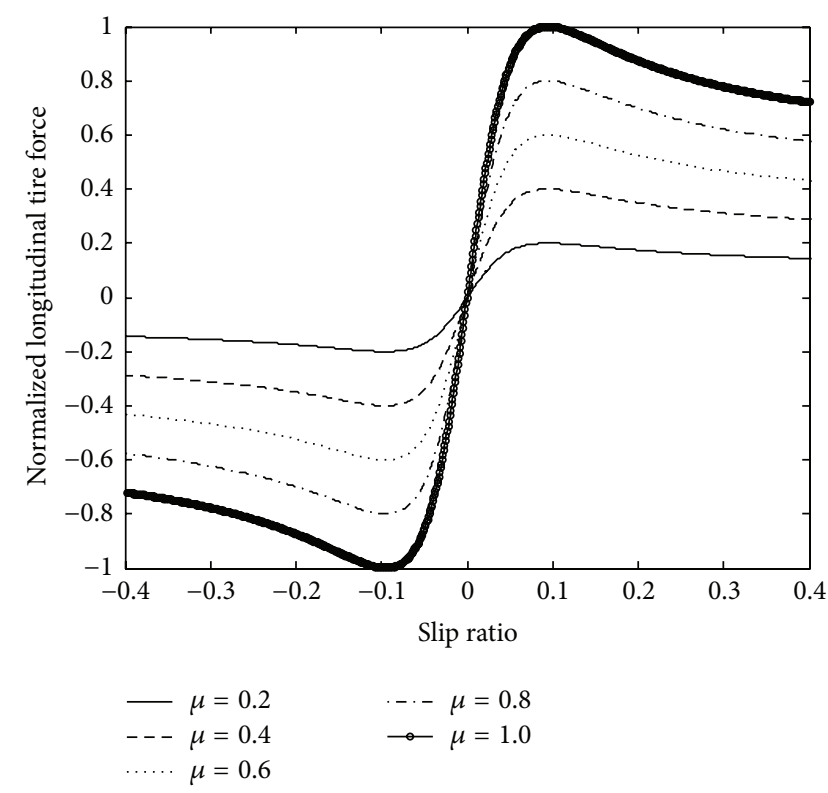

FIgURE 5: $s-\varphi$ curve under different assumed friction coefficients.

coefficient; $C_{\text {roll }}$ is the rolling resistance coefficient; and $g$ is the acceleration of gravity.

Therefore, in the ith assumed tire model as (13), the corresponding acceleration is calculated as

$$
a_{x i}=\frac{\left(F_{x i}-D_{a} v^{2}-C_{\text {roll }} m g\right)}{m} .
$$

(3) The defined deviations are

$$
E_{i}=\frac{\left|a_{x i}-a_{x}\right|}{a_{x}} \quad i=1,2,3,4,5,
$$

where $a_{x}$ is the actual acceleration which is measured by the accelerator. The conditional probability corresponding to each assumed road friction coefficient can be described as the probabilities distribution of the $E_{i}$. Assuming that the errors obey the Gaussian distribution, the likelihood function can be obtained as follows:

$$
p\left(a_{x} \mid \mu_{i}\right)=\frac{e^{-E_{i}^{2} / 2 \sigma^{2}}}{\sqrt{2 \pi} \sigma} \quad i=1,2,3,4,5,
$$

where $\sigma$ is the standard deviation.

(4) Define the a priori probability for the $i$ th assumed road friction coefficient $\mu_{i}$ as $P\left(\mu_{i}\right) . \sum_{i=1}^{5} P\left(\mu_{i}\right)=1$; according to the Bayesian theorem [35], the posterior conditional probability for $\mu_{i}$ is

$$
P\left(\mu_{i} \mid a_{x}\right)=\frac{p\left(a_{x} \mid \mu_{i}\right) P\left(\mu_{i}\right)}{\sum_{j=1}^{5} p\left(a_{x} \mid \mu_{j}\right) P\left(\mu_{j}\right)} \quad i=1,2,3,4,5 .
$$

(5) The actual road friction coefficient can be identified as follows:

$$
\mu=\sum_{i=1}^{5} \mu_{i} P\left(\mu_{i} \mid a_{x}\right) \quad i=1,2,3,4,5 .
$$




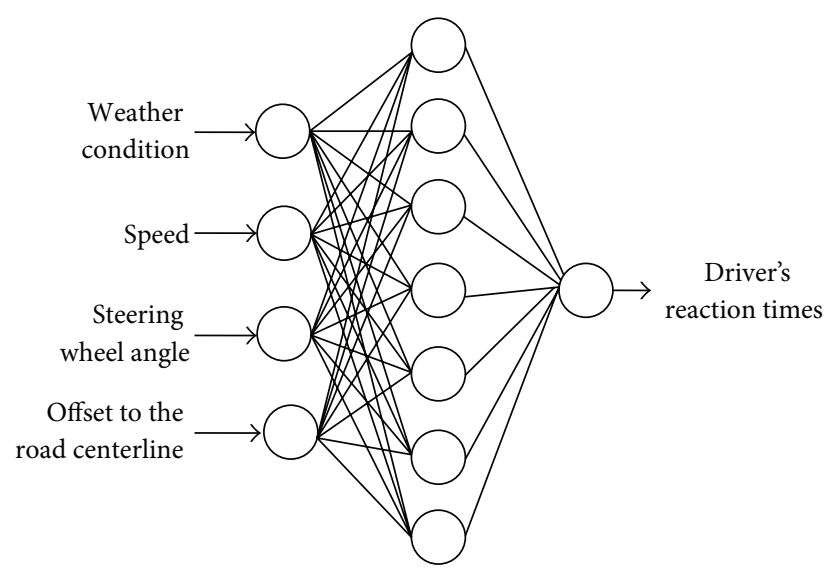

FIgURE 6: The structure of BP neural network.

3.3. Estimation of the Driver's Reaction Time. A large number of literature references and experimental results indicate that the driver's reaction time is generally $0.3 \sim 1.5 \mathrm{~s}[36,37]$. Due to the wide range of the driver's reaction time, it has a great impact on the safety distance. For example, if the speed of vehicle is $30 \mathrm{~m} / \mathrm{s}$, the calculated distances are $9 \mathrm{~m}$ and $45 \mathrm{~m}$; when the driver's reaction time are $0.3 \mathrm{~s}$ and $1.5 \mathrm{~s}$, respectively, they are $36 \mathrm{~m}$ away. The driver's reaction time is closely related to the driver fatigue, the weather condition, and the speed of vehicle. According to $[38,39]$, the steering wheel angle and the offset value to the road centerline can be used as the evaluation criteria of the driver fatigue.

However, how to mathematically model the nonlinear relationship between the driver's reaction time and these factors is a challenge. For this reason, a BP neural network-based algorithm is proposed to determine the driver's reaction time according to its general ranges.

Neural network is powerful to model nonlinear function which can be trained offline utilizing the experiment data collected in specific environment. Compared with other modeling methods, neural network has higher generalization ability to adapt to more complex environments.

As shown in Figure 6, this model has four inputs and one output. The output is the driver's reaction time. The inputs are the speed of vehicle, the steering wheel angle, the offset value to the road centerline, and the weather condition. The former three inputs can be measured by the corresponding sensors in real-time. The weather condition can be divided into four types, that is, sunny, rainy, snowy, and foggy, and can be selected beforehand. The training data is collected through experiments in different situations. The training process is offline, and the trained BP neural network is used to determine the driver's reaction time online.

There are 7 nodes in the hidden layer in the neural network. The model uses the standard BP algorithm, the transfer function from the input layer to the hidden layer is the bipolar function $f(x)=1 /\left(1+e^{-x}\right)$, and the transfer function from the hidden layer to the output layer is the linear function $f(x)=k x$. Many literature references show that the BP neural network algorithm is mature $[40,41]$, so the specific
TABLE 1: Simulation results of BP neural network model.

\begin{tabular}{lcccccc}
\hline Speed $(\mathrm{m} / \mathrm{s})$ & 10 & 15 & 20 & 25 & 30 & 35 \\
\hline Learning data $(\mathrm{s})$ & 0.33 & 0.52 & 0.76 & 0.99 & 1.13 & 1.27 \\
\hline Simulation data $(\mathrm{s})$ & 0.35 & 0.49 & 0.81 & 0.93 & 1.21 & 1.22 \\
\hline Error rate $(\%)$ & 6 & 5 & 6 & 6 & 6 & 4 \\
\hline
\end{tabular}

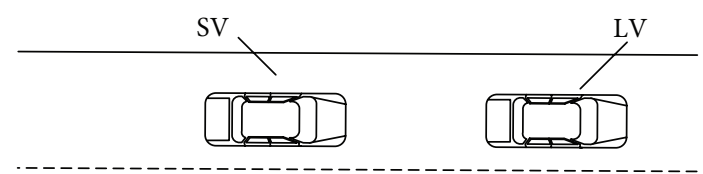

FIgURE 7: The rear-end collision scenario.

implementation of the algorithm will be not discussed in this paper.

The network weights and thresholds of the hidden layer and the output layer are obtained by using MATLAB offline training; then another set of inputs are simulated and analyzed; the results are shown in Table 1.

\section{A Variable-Threshold Method for Rear-End Collision Warning}

As shown in Figure 7, two vehicles are driving on the road, that is, the subject vehicle (SV) and the leading vehicle (LV). SV and LV constitute a rear-end collision system. The safety distance is defined as the minimum distance required to stop the SV to avoid collision; it is used to be compared with the actual distance to judge the collision danger.

A variable-threshold calculation method of the safety distance is proposed in this section. This method adopts different calculation method under different states of LV. The speeds of SV and LV are $v_{x}$ and $v_{0}$, respectively; then the relative speed is $v_{r}=v_{0}-v_{x}$. Considering the actual driving situation, the rear-end collision may occur only when $v_{r}<0$. Define that the maximum braking deceleration of SV is $a$, the brake lag time is $t_{1}$, the continuous braking time is $t_{2}$, and the real-time acceleration of LV is $a_{0}$. The safety distance $d_{s}$ can be calculated through the distance traveled by the SV minus the distance traveled by LV in period $\left(t_{1}+t_{2}\right)$ :

$$
\begin{aligned}
d_{s}= & \left(v_{x} t_{1}+v_{x} t_{2}+\frac{a t_{2}^{2}}{2}\right) \\
& -\left[v_{0}\left(t_{1}+t_{2}\right)+\frac{a_{0}\left(t_{1}+t_{2}\right)^{2}}{2}\right] .
\end{aligned}
$$

In (20), $v_{0}=v_{r}+v_{x} ; a=\mu \times g$, where $g$ is the acceleration of gravity and $\mu$ is the road friction coefficient. $a_{0}=a_{r}+a_{x}, a_{x}$ is the acceleration of SV. The brake lag time $t_{1}$ consists of three parts $[36,37]$, that is, the driver's reaction time, the braking coordination time, and the deceleration increase time. According to experience value, the braking coordination time is $0.35 \mathrm{~s}$. The deceleration increase time depends on the type of brake; the values of the hydraulic brake system and the air brake system are $0.2 \mathrm{~s}$ and $0.7 \mathrm{~s}$, respectively. The driver's reaction time, the 
road friction coefficient, and the relative acceleration are crucial parameters in (20), and the estimation algorithms are discussed in Section 3.

The continuous braking time $t_{2}$ expresses the period in which the SV maintains its maximum deceleration speed and is strongly related to the driving states of LV, that is, accelerated motion state, constant speed state, or decelerated motion state. Therefore, the value of $t_{2}$ must be calculated according to the different driving states of $\mathrm{LV}$, and then $d_{s}$ is correspondingly calculated.

(1) $a_{0}=0$; that is, LV is driving at a constant speed.

In this case, the collision only occurs when the speed of SV is greater than the speed of LV. Therefore, only this condition is considered. If the SV slows down to the speed of $\mathrm{LV}$, that is, $v_{x}=v_{0}$, and keeps a certain distance from $\mathrm{LV}$, the collision can be avoided. In this situation, SV maintains its maximum deceleration to the moment when the speed of SV is equal to that of SV; namely, $t_{2}$ can be expressed as $t_{2}=\left(v_{0}-v_{x}\right) / a=v_{r} / a$, and then the corresponding safety distance is

$$
d_{s}=-v_{r} t_{1}-\frac{v_{r}^{2}}{(2 a)}
$$

(2) $a_{0}>0$; that is, LV is accelerating.

In this situation, the collision also only occurs when the speed of SV is greater than the speed of LV. Since LV is accelerating, the speed of LV reaches to $v_{0}+a_{0} t_{1}$ at the moment when SV starts to brake. If the speed of SV can be reduced to $v_{0}+a_{0} t_{1}+a_{0} t_{2}$ after braking, the safety can be guaranteed. That is, $t_{2}=\left(v_{r}+a_{0} t_{1}\right) /\left(a-a_{0}\right)$, and the needed safety distance can be calculated as

$$
d_{s}=-v_{r} t_{1}-\frac{1}{2} a_{0} t_{1}^{2}+\frac{1}{2} \frac{\left(v_{r}+a_{0} t_{1}\right)^{2}}{a_{0}-a}
$$

(3) $a_{0}<0$; that is, LV is decelerating.

This situation is very dangerous. In this situation, only the speed of SV can be reduced to 0 after braking, that is, in stationary state, and SV keeps a certain distance to LV at the moment SV stops; the safety can be guaranteed; namely, $t_{2}=-v_{x} / a$, and then the corresponding safety distance is

$$
d_{s}=-v_{r} t_{1}-\frac{1}{2} a_{0} t_{1}^{2}+\frac{v_{x}\left(v_{0}+a_{0} t_{1}\right)}{a}-\frac{1}{2} \frac{\left(a+a_{0}\right) v_{x}^{2}}{a^{2}}
$$

This method can adaptively calculate the safety distance according to the different driving states of LV and thus can effectively avoid too large or too small safety distance. Assuming that the $d$ is the real-time distance between SV and LV, by analysis of the method, we can get the following conclusions:

(1) In the situation when $a_{0} \geq 0$ : if $v_{r} \geq 0$, the collision does not occur, if $v_{r}<0$, the collision does not occur as long as the braking is performed in the period when $d \geq d_{s}$.

(2) In the situation when $a_{0}<0$, the collision does not occur if the braking is performed in the period when $d \geq d_{s}$.

\section{Simulations}

The proposed strategy was first evaluated through extensive simulations, which were performed under typical driving scenarios using CarSim in MATLAB/Simulink. Due to the experimental condition limitation and the safety concern, many algorithms are difficult to be verified in real experiments, that is, the road friction coefficient estimation algorithm and the variable-threshold method. Since the real experiments will be described in the next section, the simulations were only used to verify the road friction coefficient estimation algorithm and the variable-threshold method.

5.1. Performance of the Road Friction Coefficient Estimation Algorithm. The proposed algorithm can be apt for both high and low slip ratios. However, the high ratio scenario is hard to achieve in experiment, and the low friction coefficient scenario is dangerous for the driver. Therefore, the effectiveness and feasibility of the proposed algorithm in extreme conditions are firstly verified by simulation.

The proposed algorithm is validated in high and low slip ratios conditions with the tire-road friction coefficient changed from 0.8 to 0.2 , and the estimation results are compared with the conventional slip-slope algorithm. Simulation results show that the proposed algorithm can be applied to both high and low slip ratios and can quickly respond to the change of road condition with high accuracy.

5.1.1. Simulation at Low Slip Ratios Condition. Figures 8 and 9 are simulation results in the low ratios condition. The figures show that the values of slip ratio are small, and the proposed algorithm can quickly identify the road friction coefficient with high accuracy; the error is less than 0.1. From Figure 8, the proposed algorithm can converge to the true value within $2 \mathrm{~s}$ when the tire-road friction coefficient jumps, which can meet the real-time requirements.

5.1.2. Simulation at High Slip Ratios Condition. The conventional slip-slope algorithm is no longer suitable for the high slip ratios condition because the relationship between $s$ and $\varphi$ is no longer linear. Figures 10 and 11 are simulation results in the high slip ratios condition. The figures show that the slip-slope algorithm produces a great error. The proposed algorithm can quickly identify the road friction coefficient with high accuracy at high slip ratios and can quickly respond to the change of road condition.

5.2. Evaluation of the Variable-Threshold Method. The real road test for warning method may be dangerous in some cases, especially in the extreme conditions such as the short distance between LV and SV or the emergency braking situation of LV. Therefore, the proposed warning method was firstly verified through simulation. Five cases are set up to emulate the typical rear-end collision warning conditions. To evaluate the effect of the proposed method, the widely used kinematics-based methods, that is, Mazda method, Honda method, and Berkeley improved method, are also investigated for comparison. It should be noted that the simulation 


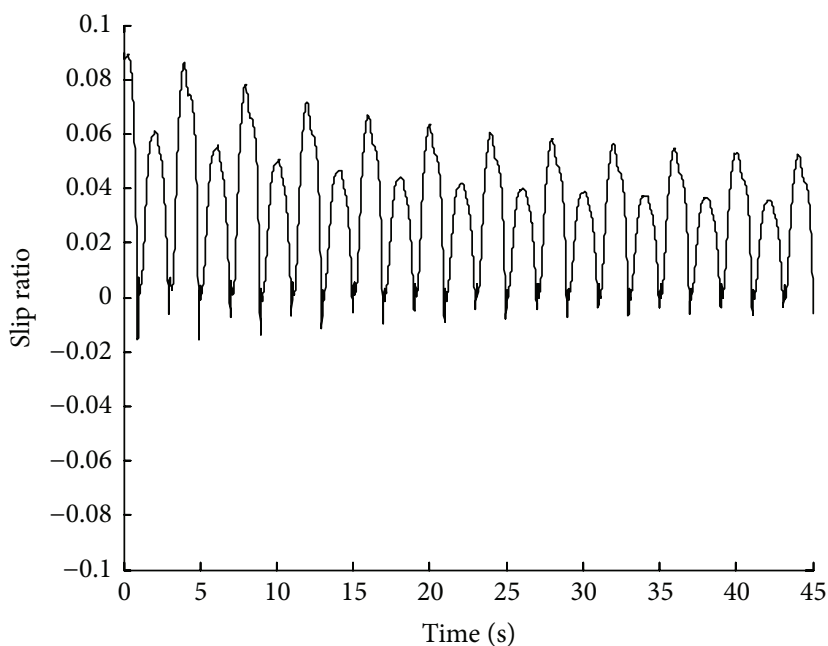

(a)

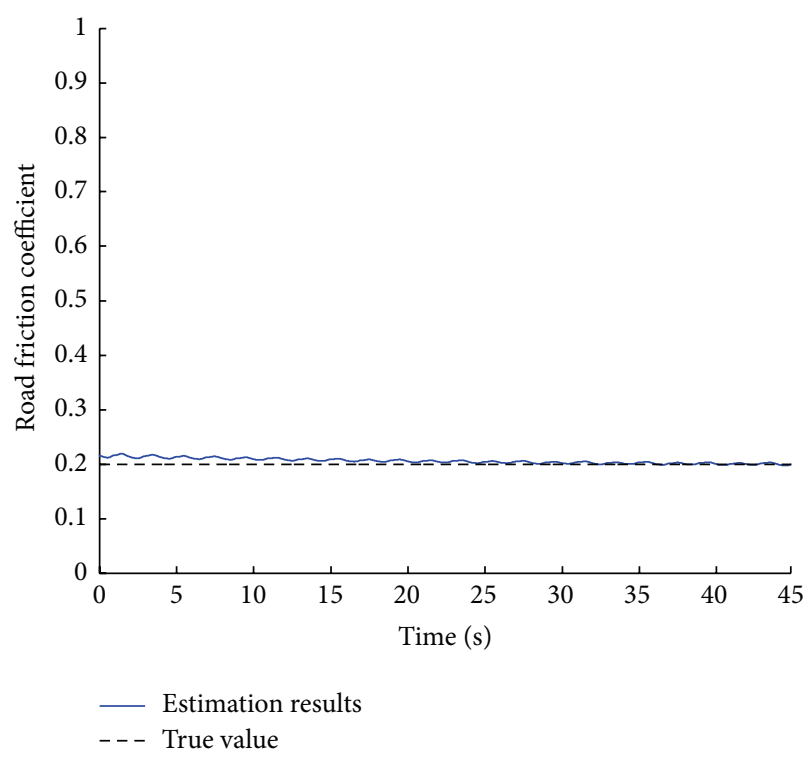

(b)

FIGURE 8: Simulation results of low slip ratios. (a) Slip ratio. (b) Tire-road friction coefficient.

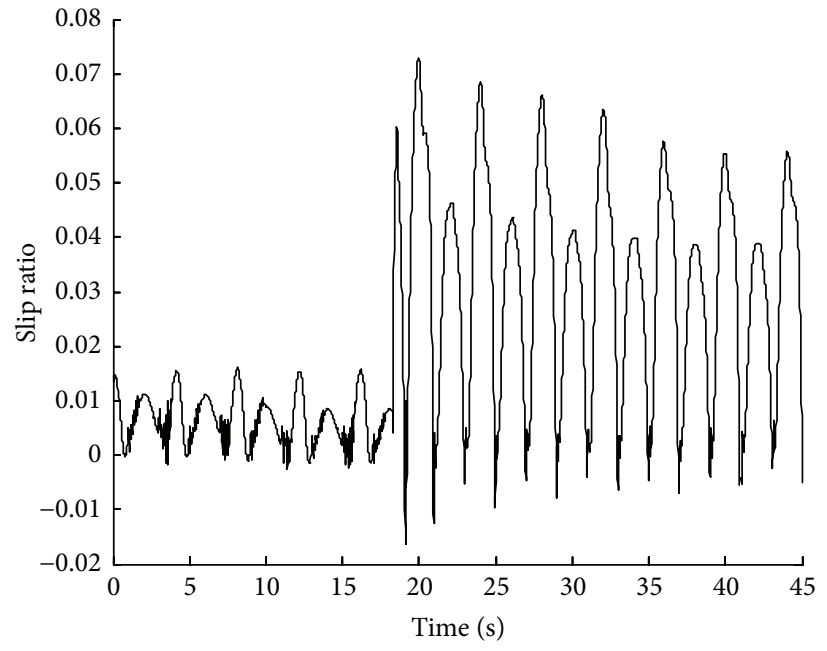

(a)

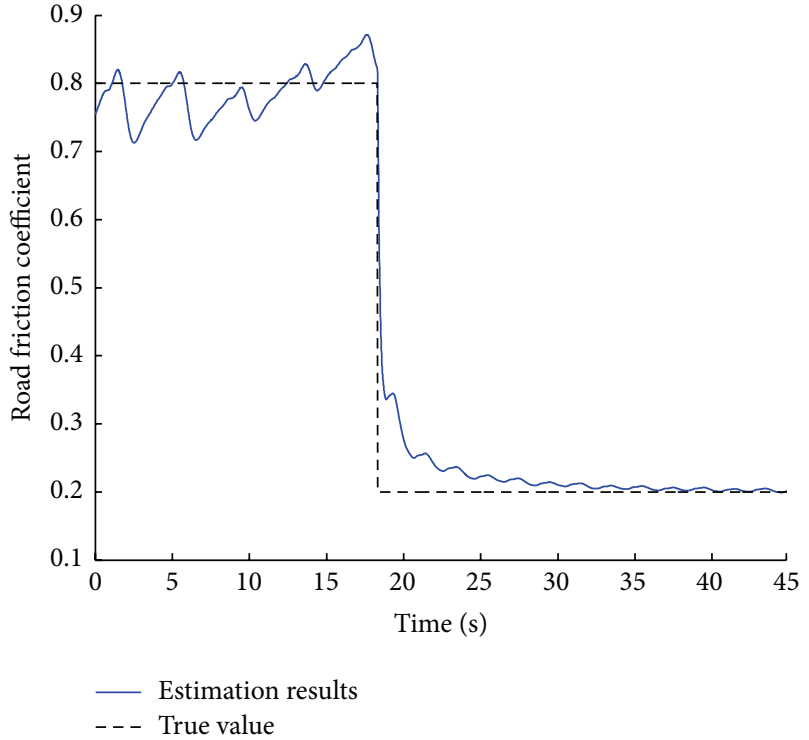

(b)

FIGURE 9: Simulation results of low slip ratios with friction coefficient changed. (a) Slip ratio. (b) Tire-road friction coefficient.

only aims to verify the proposed variable-threshold method itself and does not consider the change of parameters.

Assume that the driver's reaction time is $0.75 \mathrm{~s}$ and the road friction coefficient is 0.6 . The five cases are set up as follows:

(1) LV and SV drive at constant speed, and the speeds of LV and SV are $100 \mathrm{~km} / \mathrm{h}$ and $120 \mathrm{~km} / \mathrm{h}$, respectively. In the initial moment, the distance between LV and $\mathrm{SV}$ is $200 \mathrm{~m}$.

(2) In the initial moment, the distance between LV and $\mathrm{SV}$ is $150 \mathrm{~m}$, and the speeds of LV and SV are equal, that is, $90 \mathrm{~km} / \mathrm{h}$. Then $\mathrm{LV}$ drives at constant speed and SV starts to accelerate. The acceleration of SV is $2 \mathrm{~m} / \mathrm{s}^{2}$.

(3) The initial speeds of LV and SV are equal, that is, $110 \mathrm{~km} / \mathrm{h}$; then SV drives at constant speed and LV starts to decelerate. The initial distance between LV and $\mathrm{SV}$ is $150 \mathrm{~m}$. The deceleration of $\mathrm{LV}$ is $-2 \mathrm{~m} / \mathrm{s}^{2}$.

(4) LV and SV drive at constant speed in the initial moment, that is, $110 \mathrm{~km} / \mathrm{h}$, and then the emergency brake of $\mathrm{LV}$ is activated. The initial distance between $\mathrm{LV}$ and $\mathrm{SV}$ is $150 \mathrm{~m}$. The deceleration of $\mathrm{LV}$ is $-7 \mathrm{~m} / \mathrm{s}^{2}$. 


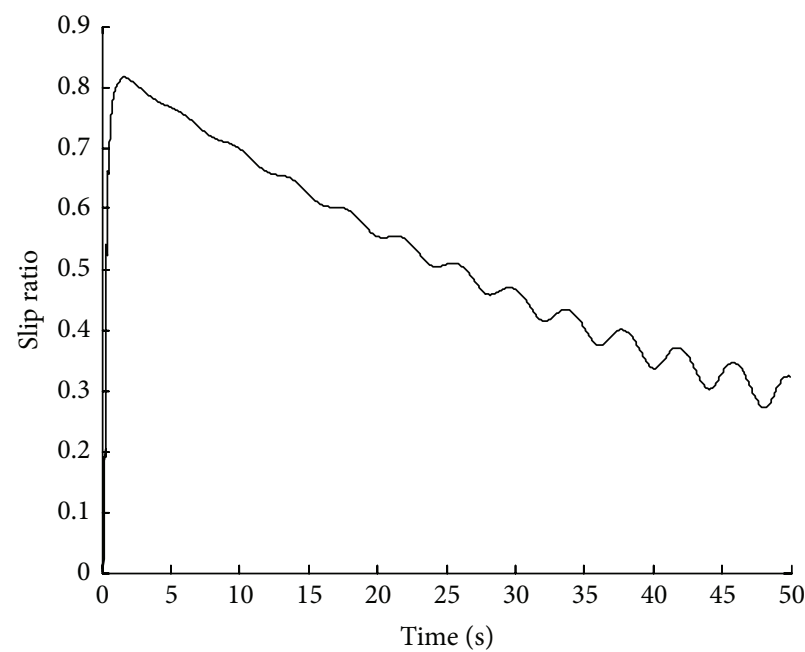

(a)

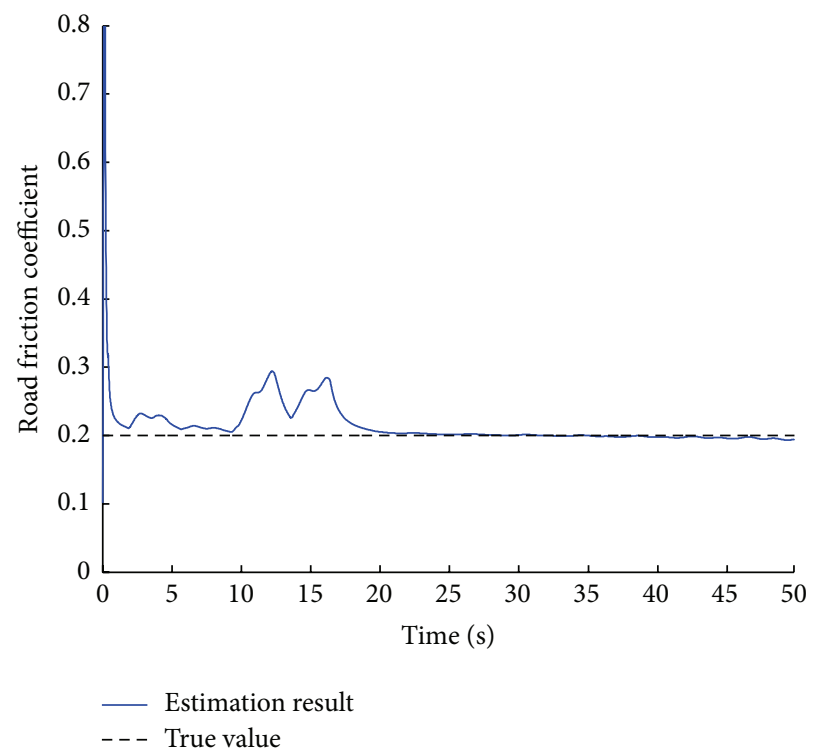

(b)

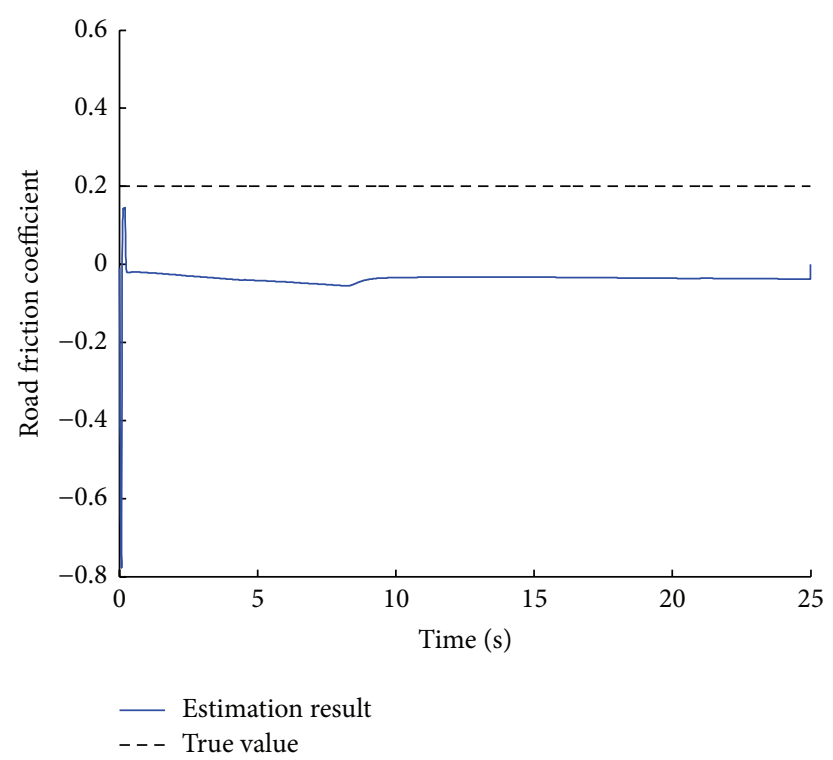

(c)

Figure 10: Simulation results of high slip ratios. (a) Slip ratio. (b) Friction coefficient estimated by the proposed algorithm. (c) Friction coefficient estimated by the slip-slope method.

(5) LV and SV drive at constant speed in the initial moment, that is, $110 \mathrm{~km} / \mathrm{h}$, and then the $\mathrm{LV}$ suddenly stops due to the emergency situations (e.g., LV hits the obstacle). The initial distance between LV and SV is $150 \mathrm{~m}$, and then the speed of LV suddenly becomes 0 .

For the four methods, that is, proposed variable-threshold method, Mazda method, Honda method, and Berkeley improved method, Figure 12 shows the simulation results in the five cases. It should be noted that the Honda method and Berkeley improved method employ two level threshold, that is, the early warning distance and the brake warning distance.

In Figure 12, the lines with different colors represent the safety distances which are calculated by different methods in real-time, and the black dotted vertical lines are the corresponding warning times.

The main advantage of the variable-threshold method is the appropriate value of the safety distance, that is, neither too large nor too small. If the safety distance is too large, the corresponding warning time is too early and may interfere with normal driving operation (i.e., false positive). If the safety distance is too small, the corresponding warning time is too late and the reaction time for driver is not sufficient to avoid collision (i.e., false negative).

Figures 12(a) and 12(b) indicate the common cases in highway which are suitable for the braking warning of the Berkeley improved method and the Honda method, respectively. The calculation result of proposed strategy is 


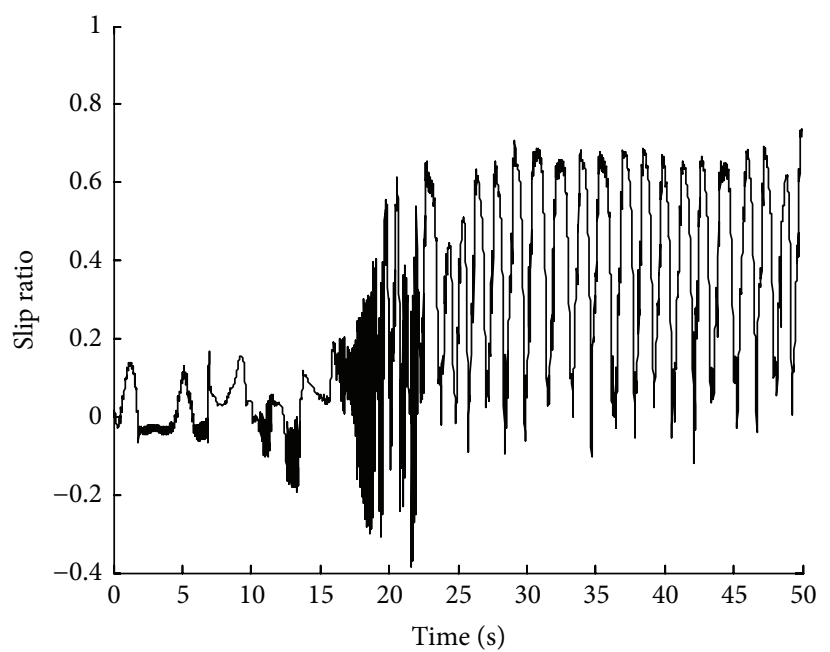

(a)

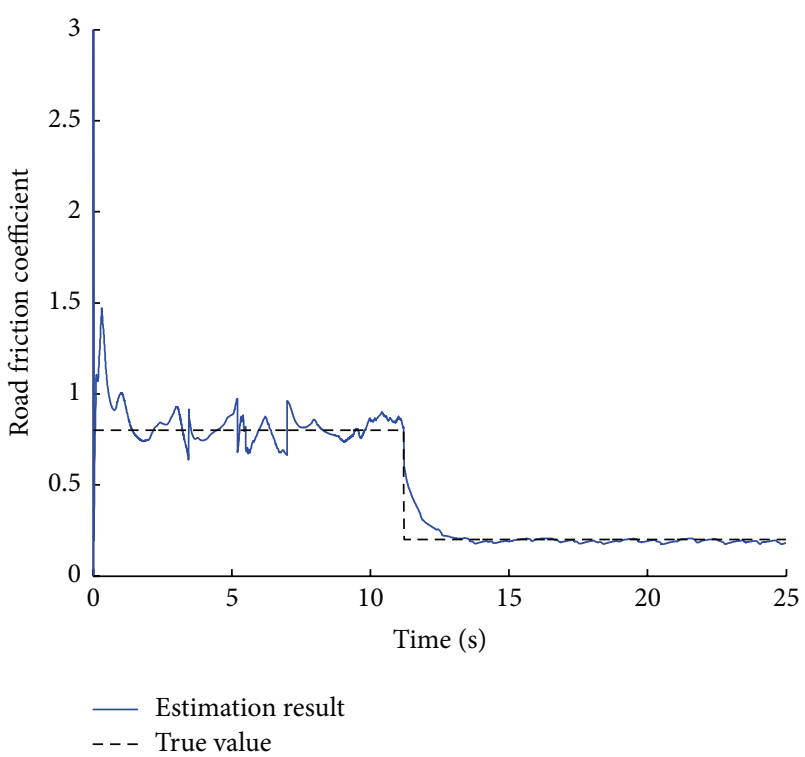

(b)

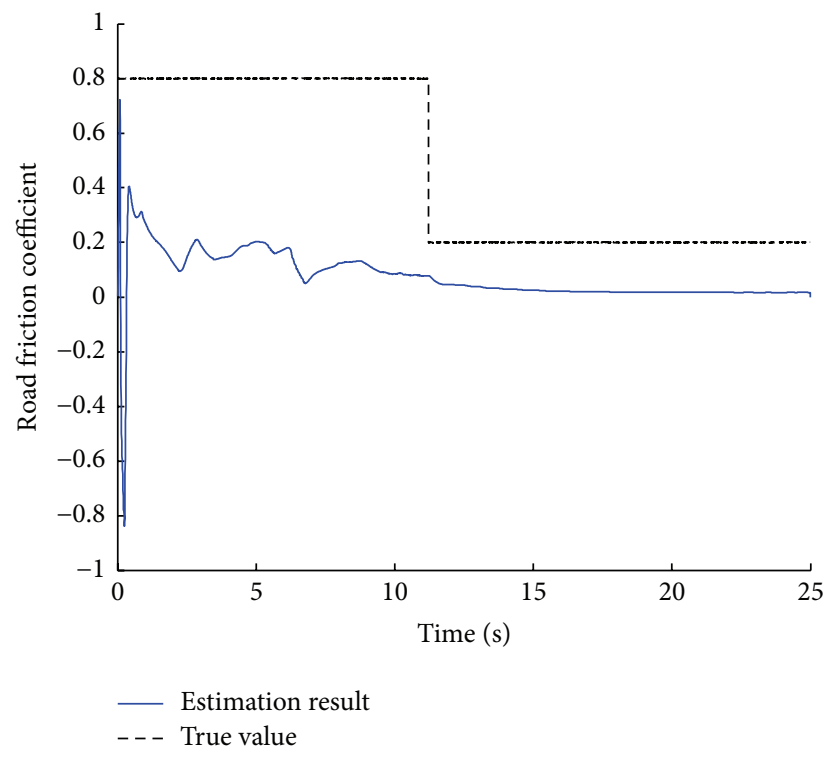

(c)

FIGURE 11: Simulation results of high slip ratios with friction coefficient changed. (a) Slip ratio. (b) Friction coefficient estimated by the proposed algorithm. (c) Friction coefficient estimated by the slip-slope method.

approximate to the results of these two methods. It can be verified that our method can adapt to different conditions. Other methods such as Mazda method lead to too early warning and may interfere with normal driving operation.

Case (3) simulates a typical dangerous condition in highway that LV and SV drive at the same speed and keep a safety distance, and then LV brakes with a deceleration of $-2 \mathrm{~m} / \mathrm{s}^{2}$. From Figure 12(c), we can find that the warning time estimated by the proposed method is about $6.6 \mathrm{~s}$, which is approximate to the early warning time of Honda method and is obviously different from that of other methods. The main reason is that this case is similar to the early warning scenario defined by Honda method, and other methods are unable to adapt to this case. If the driver starts to brake at $6.6 \mathrm{~s}$, the actual distance between LV and SV is $15.68 \mathrm{~m}$ when the vehicles are in the safe state. The proposed strategy can avoid the collision accident and leave a certain safety distance between SV and LV. Taking Mazda method as an example, the corresponding warning time is about $5.1 \mathrm{~s}$. If the driver starts to brake at $5.1 \mathrm{~s}$, the actual distance between LV and SV is $42.31 \mathrm{~m}$ when the vehicles are in the safe state. This distance is too long and may interfere with normal driving operation.

Case (4) simulates a more dangerous condition that LV and SV drive at the same speed and keep a safety distance, and then LV brakes with a high deceleration of $-7 \mathrm{~m} / \mathrm{s}^{2}$ due to the emergency situation. From Figure 12(d), it can be seen 


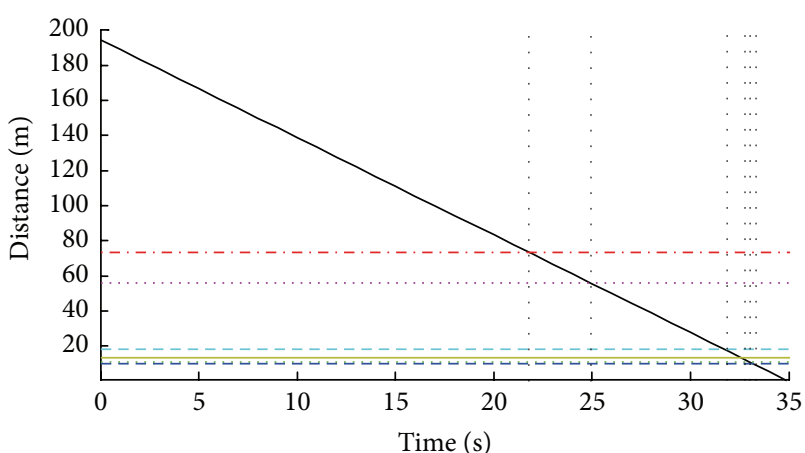

- Distance between LV and SV

...... Safety distance by using Mazda algorithm

-. Early warning distance by using Honda algorithm

_ Brake warning distance by using Honda algorithm

-.. - Early warning distance by using Berkeley improved algorithm

.... Brake warning distance by using Berkeley improved algorithm

-. - Safety distance by using proposed algorithm

(a)

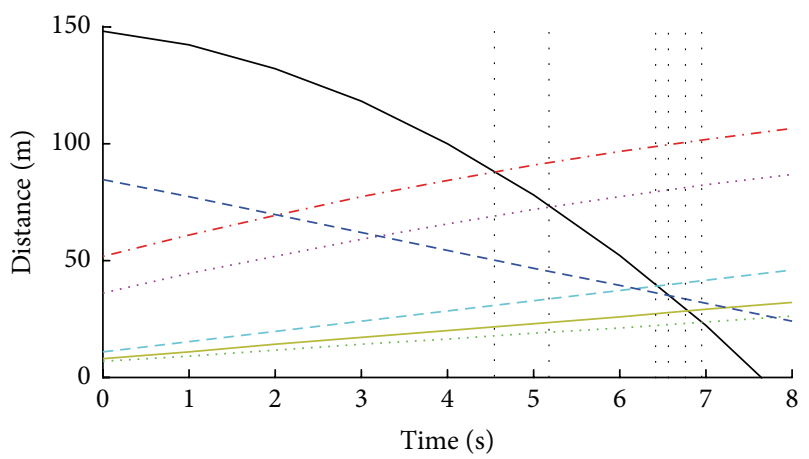

- Distance between LV and SV

...... Safety distance by using Mazda algorithm

-. - Early warning distance by using Honda algorithm

_ Brake warning distance by using Honda algorithm

...- Early warning distance by using Berkeley improved algorithm

...... Brake warning distance by using Berkeley improved algorithm

-.- Safety distance by using proposed algorithm

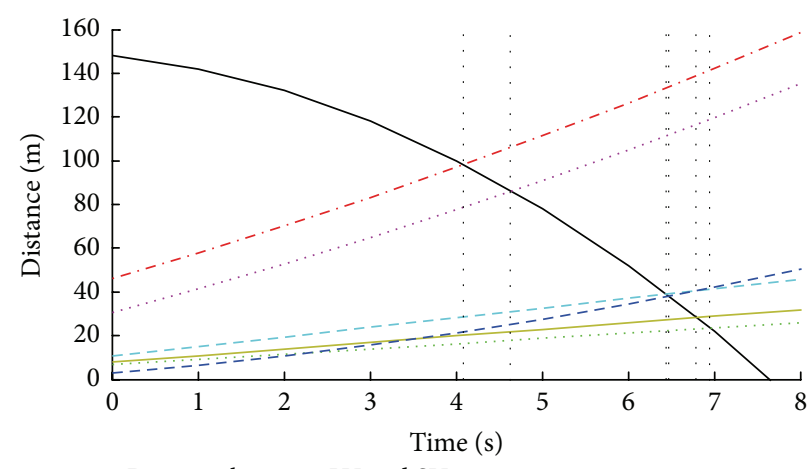

- Distance between LV and SV

...... Safety distance by using Mazda algorithm

- Early warning distance by using Honda algorithm

- Brake warning distance by using Honda algorithm

-... - Early warning distance by using Berkeley improved algorithm

... Brake warning distance by using Berkeley improved algorithm

- - - Safety distance by using proposed algorithm

(b)

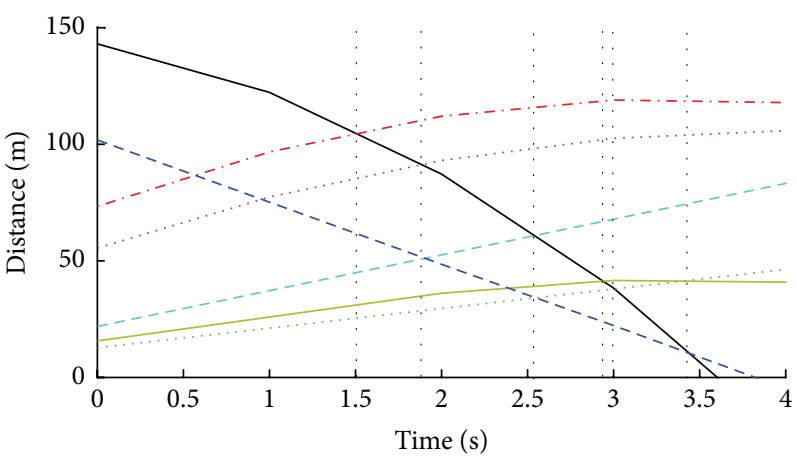

- Distance between LV and SV

..... Safety distance by using Mazda algorithm

- . - Early warning distance by using Honda algorithm

_ Brake warning distance by using Honda algorithm

-.. - Early warning distance by using Berkeley improved algorithm

.... Brake warning distance by using Berkeley improved algorithm

-.. Safety distance by using proposed algorithm

(c)

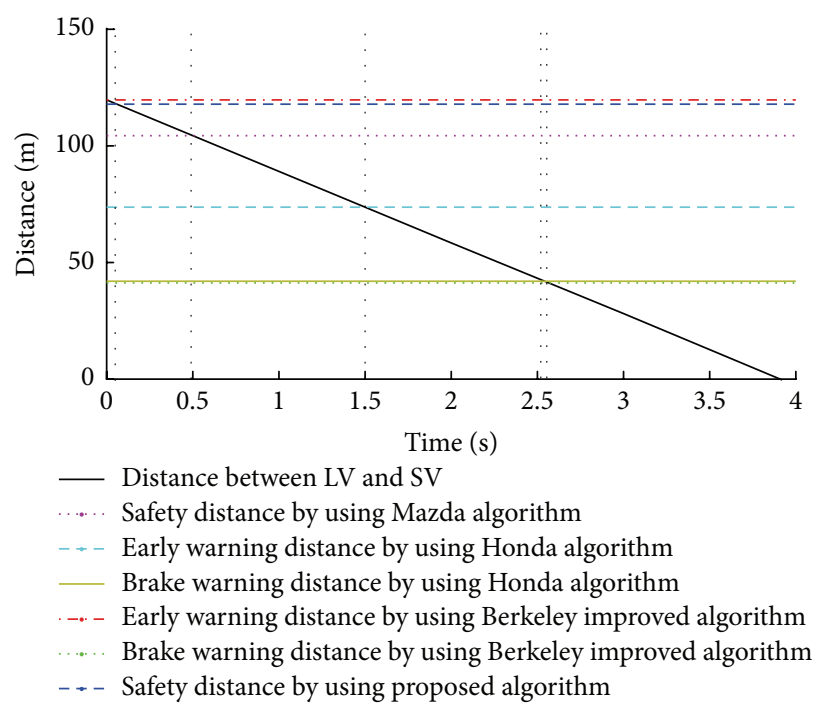

(e)

FIgURE 12: The simulation results of variable-threshold method. 
that the warning time estimated by the proposed method is about $3.4 \mathrm{~s}$, which is approximate to the brake warning time of the Berkeley improved method. The phenomena can be attributed the similar reasons as case (3). The warning times of other methods are too early and then may lead to false negative.

Case (5) is the most dangerous condition in highway which is suitable for the early waning of the Berkeley improved method and the Mazda method. From Figure 12(e), we can find that the warning time estimated by the proposed method is approximate to the results of the early waning of Berkeley improved method, that is, about $0.1 \mathrm{~s}$. If the driver starts to brake at this moment, the actual distance between $\mathrm{LV}$ and SV is $11.28 \mathrm{~m}$ when the vehicles are in the safe state. The proposed strategy can avoid the collision accident and leave a certain safety distance between SV and LV. Compared with other methods such as brake warning of Honda method, the warning time is about $2.5 \mathrm{~s}$. If the driver starts to stop at $2.5 \mathrm{~s}$, SV will hit LV and thus the collision occurs.

Through the above analysis, it is clear that each of the conventional methods has its specific case and can work well in this case. However, one method is unable to adapt to other cases and will lead to false negative or false positive in other cases. The proposed variable-threshold method can combine the advantages of these conventional methods; that is, it can adjust the calculation method of safety distance according to the driving state of LV to make the method adapt to various scenarios. In a particular scenario, the safety distance calculated by the proposed method is approximate to that of the corresponding method which can adapt to the certain scenario.

In addition, the proposed method has other advantages; for example, it can accommodate to different road conditions and driver's states; that is, the road friction coefficient and driver's reaction time can be estimated in real-time to further improve the adaptability under different driving scenarios. These characteristics are not verified by the simulation and will be validated through the real experiments in the next section.

\section{Experimental Results}

To further verify the performance of the proposed warning strategy in practice, experiments were conducted on a Buick Sail SRV vehicle. It was equipped with many low-cost sensors as shown in Figure 1. The experiments were carried out on the campus. During the experiments, all sensor data were collected, and then the warning strategy was evaluated by using the logged data. For brevity, only two tests are shown here as an example because similar conclusions can be drawn by other tests.

6.1. Experiment 1. In this experiment, the test duration is $50 \mathrm{~s}$, and the experimental road contains the dry and wet asphalt roads. Parts of the signals collected by the sensors are shown in Figure 13 to illustrate the driving status.

\subsubsection{Performance of Key Parameters Estimation Algorithms}

(1) Performance of the Relative Acceleration Estimation Algorithm. Figure 14 gives the estimation processes and results of the relative acceleration. For comparison and analysis, the estimation result by the Euler forward difference method is also investigated.

It can be seen that the relative acceleration estimation result based on the Euler difference method (Figure 14(c)) has great error, and there are large amounts of data loss and latency. But the curve is in line with the changing trend of the relative speed. The relative acceleration preliminary estimation result based on Kalman filter (the blue dashed line in Figures 14(a) and 14(d)) does not meet the relative speed changing trend at some point, but there are no data loss and latency. The second stage estimation of relative acceleration (the purple solid line in Figures 14(a) and 14(d)) combines the advantages of both methods. It is in accordance with the changing trend of the relative speed and has high accuracy. For example, the relative speed declines in 27-30 seconds, so the corresponding relative acceleration should be less than 0 . But the preliminary estimation result of the relative acceleration is more than 0 before correction, and this problem is solved by using the correction algorithm in the second stage. To obtain the reference value of relative acceleration to verify the effectiveness of the proposed algorithm, two accelerators are installed on the LV and SV to measure their accelerations, respectively. Figure 14(d) shows comparison of the reference value and the estimation results. Figure 14(e) illustrates the estimation errors.

Obviously the corrected value has smaller error and better accuracy, and it is consistent with the change of the relative speed. Therefore, the proposed algorithm can provide more accurate relative acceleration information and then can improve the performance of subsequent warning method.

(2) Performance of Road Friction Coefficient and Driver's Reaction Time Estimation Algorithms. Figure 15 shows the estimation results of the road friction coefficient and the driver's reaction time. From Figure 15(a), we can find that the road friction coefficient changes from about 0.4 to about 0.7 when the vehicle drives from the wet asphalt road to the dry asphalt road, which are approximate to the theoretical values. It can be seen in Figure 15(b) that the driver's reaction time can change according to the different driving situations.

6.1.2. Performance of Variable-Threshold Warning Method. To evaluate the performance of the proposed variable-threshold method, other commonly used methods, such as Mazda method, Honda method, and Berkeley improved method, are also investigated for comparison. Figure 16 illustrates the real-time distance between LV and SV and the estimated safety distances from proposed and other commonly used methods.

From Figure 16, we can find that the warning is issued to warn the driver only in the 27-30 s. In the periods, the relative speed is less than 0; that is, the speed of SV is far greater than that of LV. Meanwhile, the distance between LV and SV is short and the speed of SV is large. Therefore, the warning 


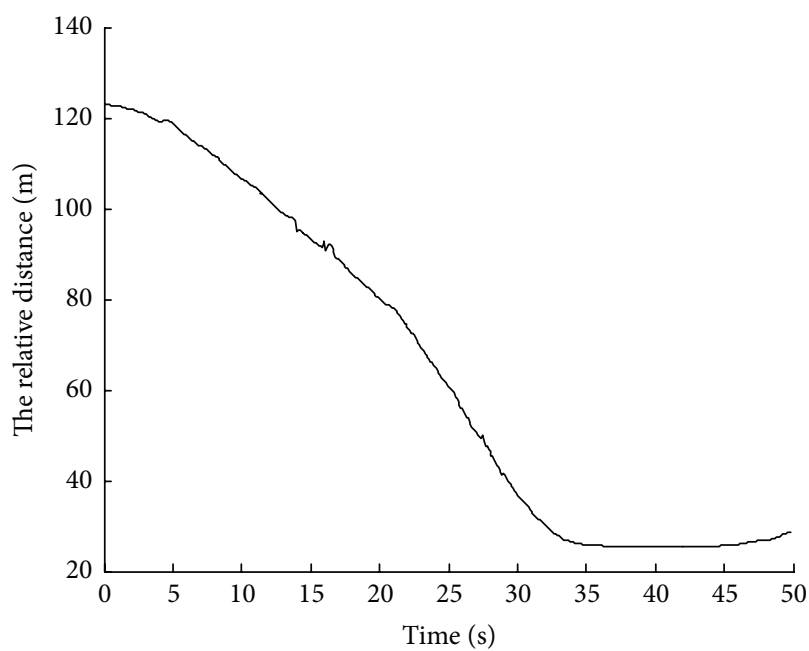

(a)

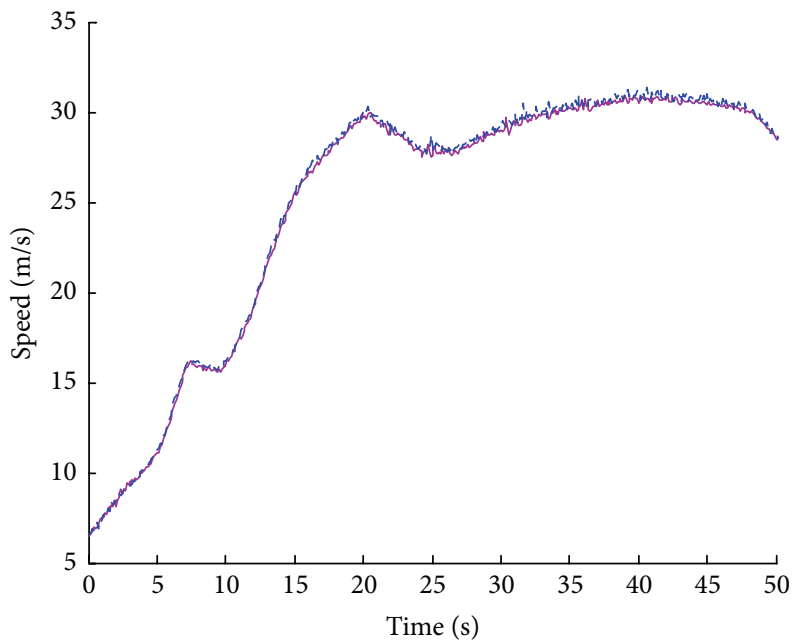

- - Single front wheel speed

(c)

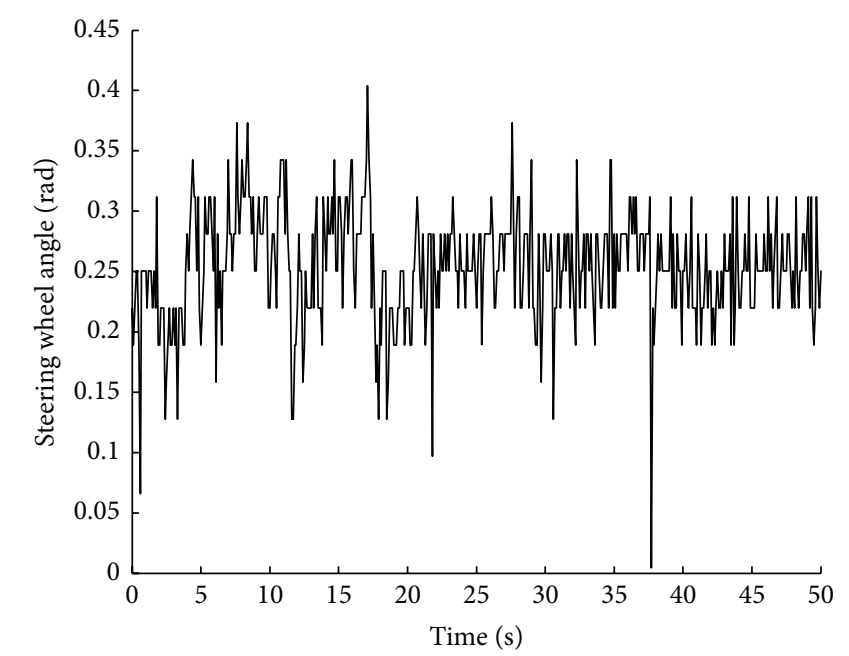

(e)

FIgURE 13: The information collected by the sensors in experiment 1. (a) The distance between LV and SV. (b) The relative speed. (c) The speed and the single front wheel speed of SV. (d) The acceleration of SV. (e) The steering wheel angle of SV. 


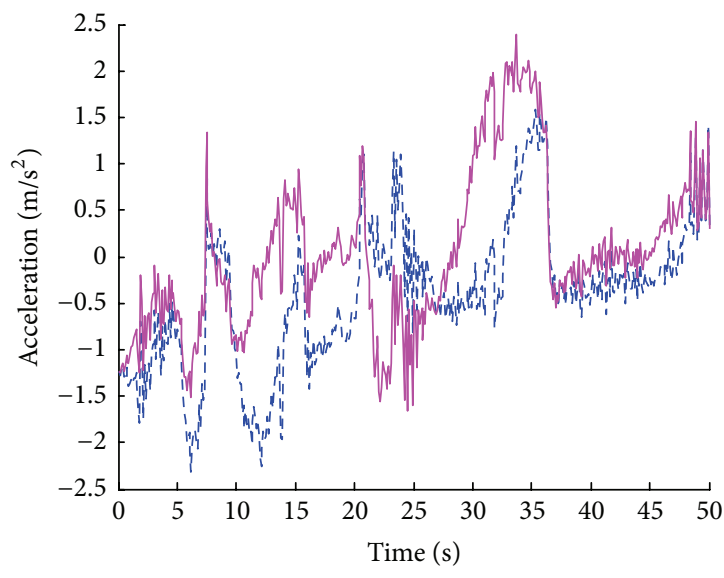

- - - Before correction

- After correction

(a)

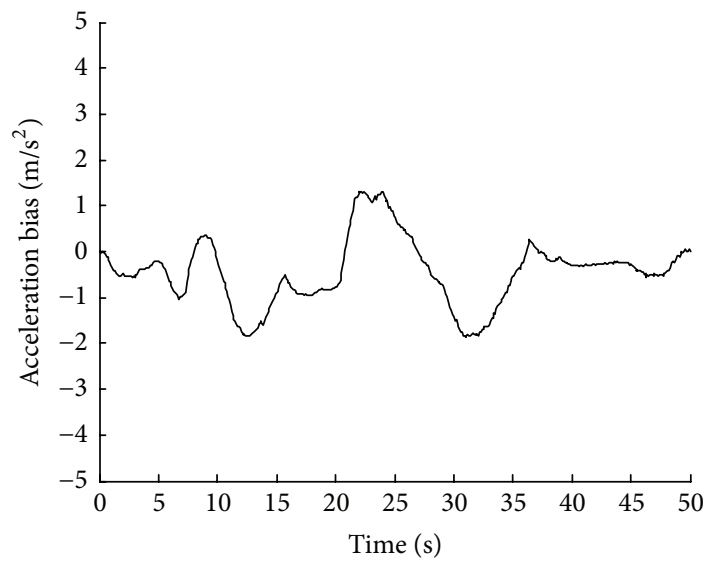

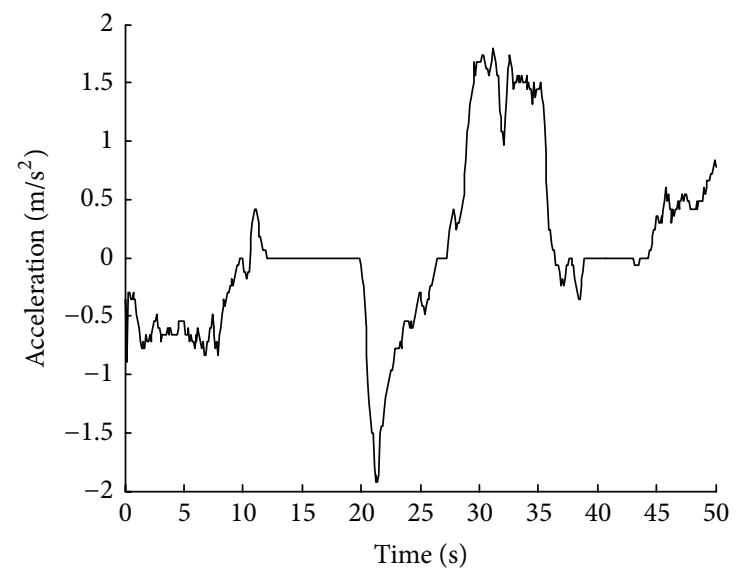

(b)

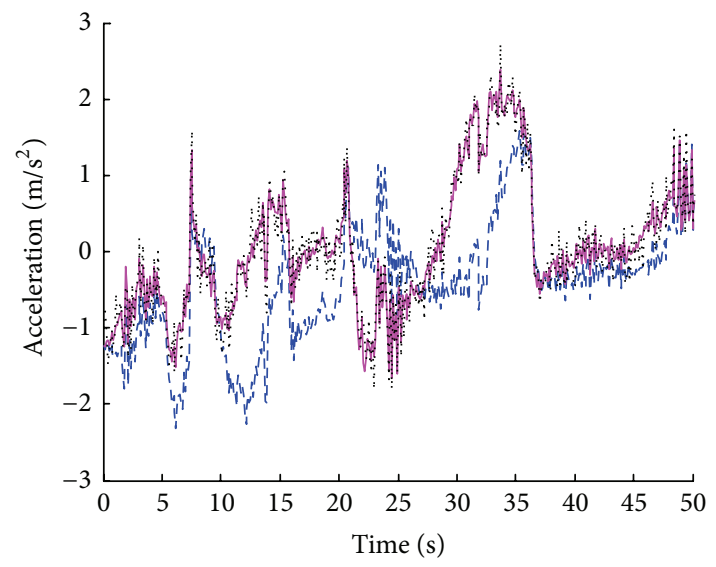

- - Before correction

After correction

True value

(d)

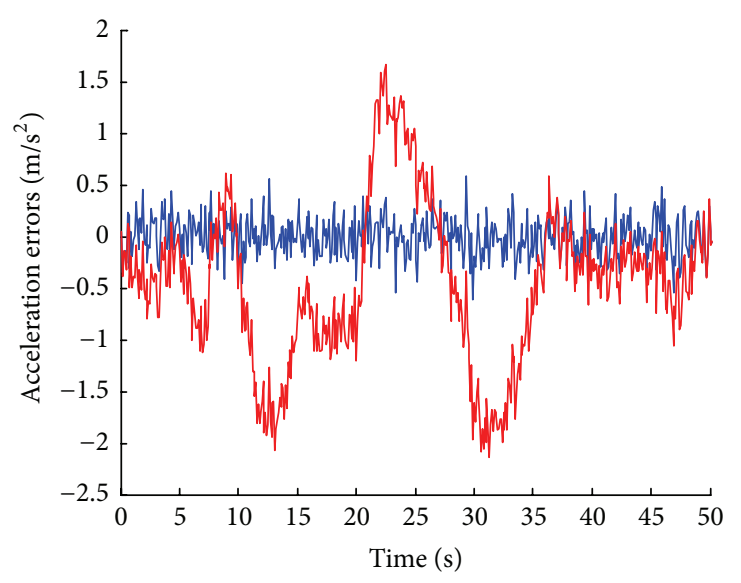

- After correction

— Before correction

(e)

FIGURE 14: The estimation of the relative acceleration. (a) The preliminary and final estimation results by the proposed two-stage algorithm. (b) The estimation result by the Euler forward difference method. (c) The preliminary estimation bias. (d) The estimation results and the reference values. (e) The preliminary and final estimation errors. 


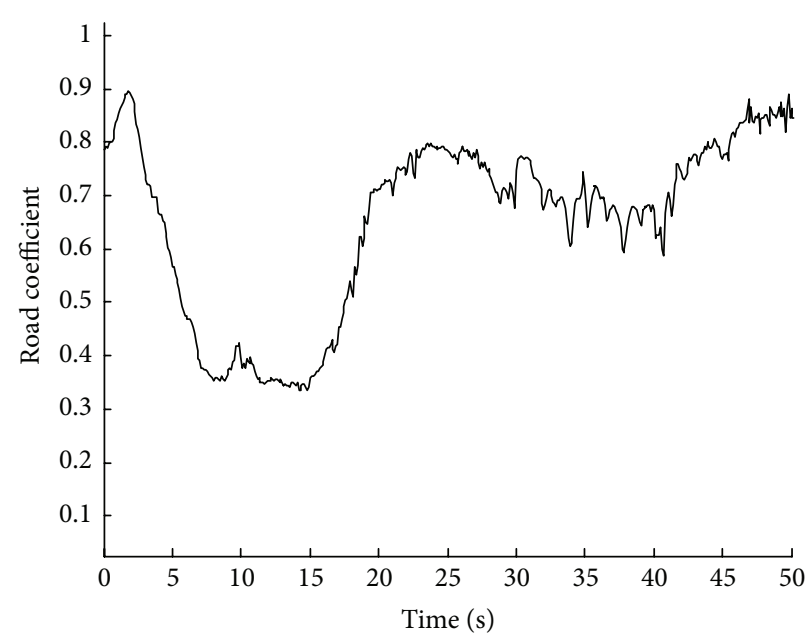

(a)

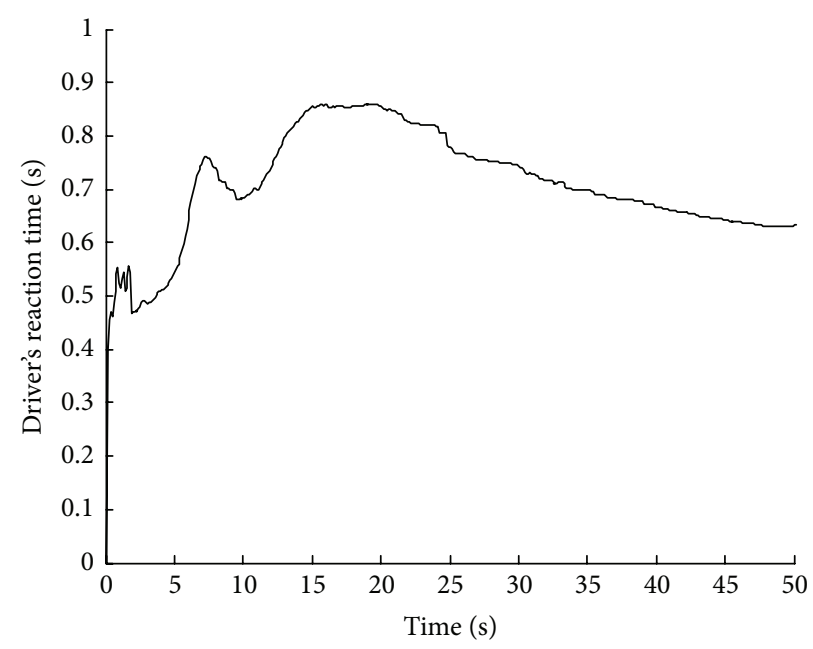

(b)

FIGURE 15: The estimation results of key parameters. (a) The road friction coefficient. (b) The driver's reaction time.

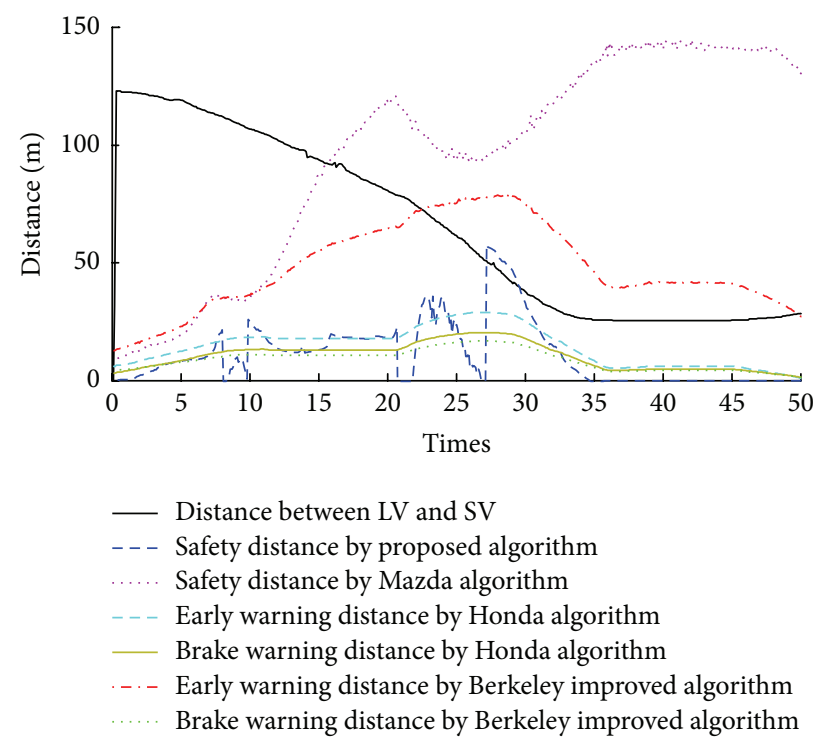

FIgURE 16: The experimental results of safety distance calculation.

time has a good agreement with the actual situation and the intuitive sense of the driver.

In this experiment, the warning times of two methods, that is, Mazda method and the early warning of the Berkeley improved algorithm, are earlier than that of the propose strategy, and the warning is not activated in other methods.

Assuming that SV and LV keep their current state after $27 \mathrm{~s}$, by analyzing the relative movement and calculating the time to collision (TTC), we can deduce the conclusion that the collision may occur within $5 \mathrm{~s}$. Therefore, the warning is necessary in this situation, and the other methods which have no warning may lead to false negative. Taking the warning time of the Mazda method, that is, $15 \mathrm{~s}$, as example, the TTC is about $25 \mathrm{~s}$; namely, assuming that SV and LV keep their current state after $15 \mathrm{~s}$, the collision may occur after $25 \mathrm{~s}$.
Obviously the warning time is too early and may lead to false positive.

The experiment contains different driving scenarios, that is, the acceleration and deceleration of LV and SV, and the road friction coefficient changes. Other methods are only suitable for a specific case and assume that the road friction coefficient is constant. The proposed method can combine the advantages of these conventional methods and can estimate the road friction coefficient in real-time, which can obviously improve the adaptability.

From the above analysis, we can get the similar conclusions as the simulation results in Section 5.2; that is, compared with other methods, the proposed method has some advantages; for example, it can adapt to various driving states of LV, various road conditions, and various driver's states.

6.2. Experiment 2. Another experiment was carried out on the dry asphalt road. The test duration is $40 \mathrm{~s}$, and the experiment also contains different driving scenarios, that is, the acceleration and deceleration of LV and SV. Parts of the signals collected by sensors are shown in Figure 17.

Figure 18 shows the key parameters estimation results of experiment 2 . The estimation results have a good agreement with the actual situation.

From Figures 18(a) and 18(b), we can find that the proposed relative acceleration estimation algorithm has high accuracy; that is, the estimation error is less than $0.3 \mathrm{~m} / \mathrm{s}^{2}$. The estimated road friction coefficient is about 0.7 , which is approximate to the theoretical value of the dry asphalt road. The driver's reaction time is varying with the change of driving status and eventually converges to about $0.5 \mathrm{~s}$.

Figure 19 shows the real-time distance between LV and SV (black solid line) and the estimated safety distances by proposed strategy (blue dashed line). It is clear that there is no condition needs warning. It can be seen in the calculation formula of safety distance that the distance can be modified to adapt to the actual road condition if the actual road friction 


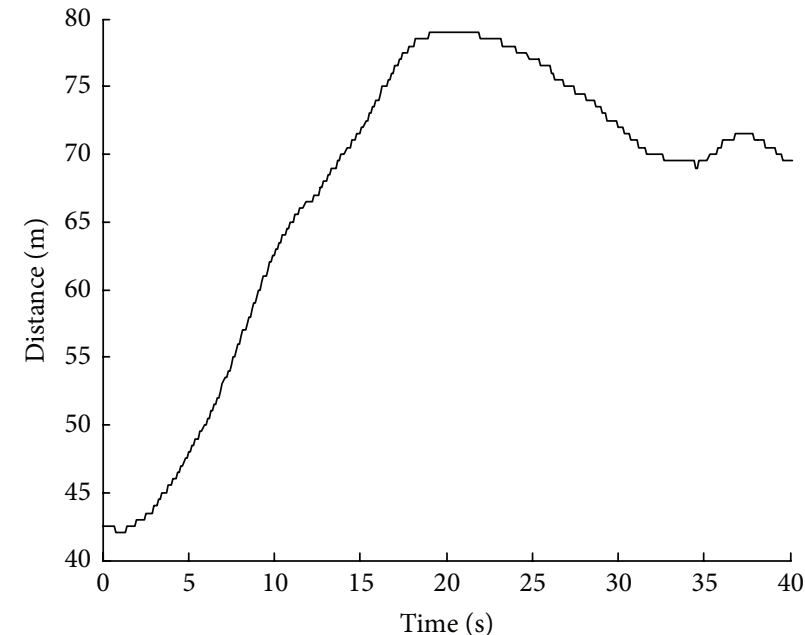

(a)

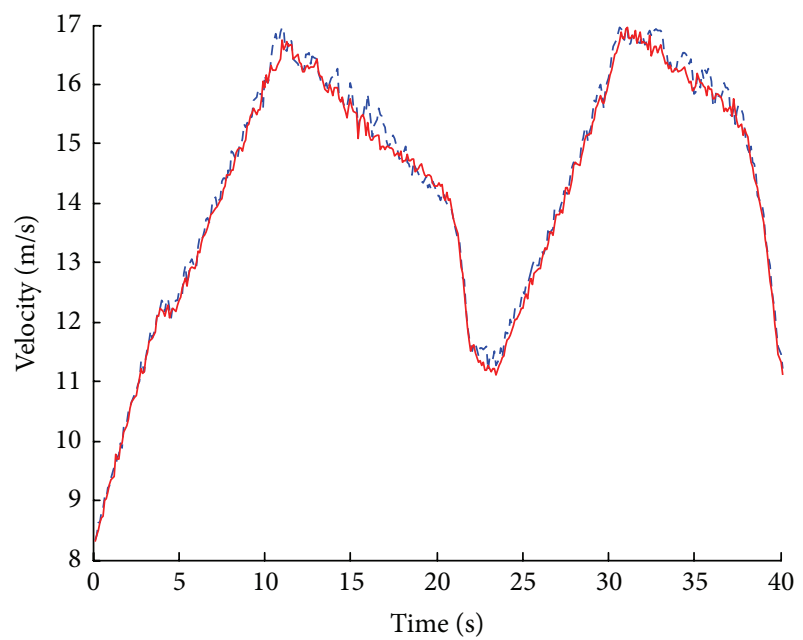

- - - Single front wheel speed

__ Vehicle speed

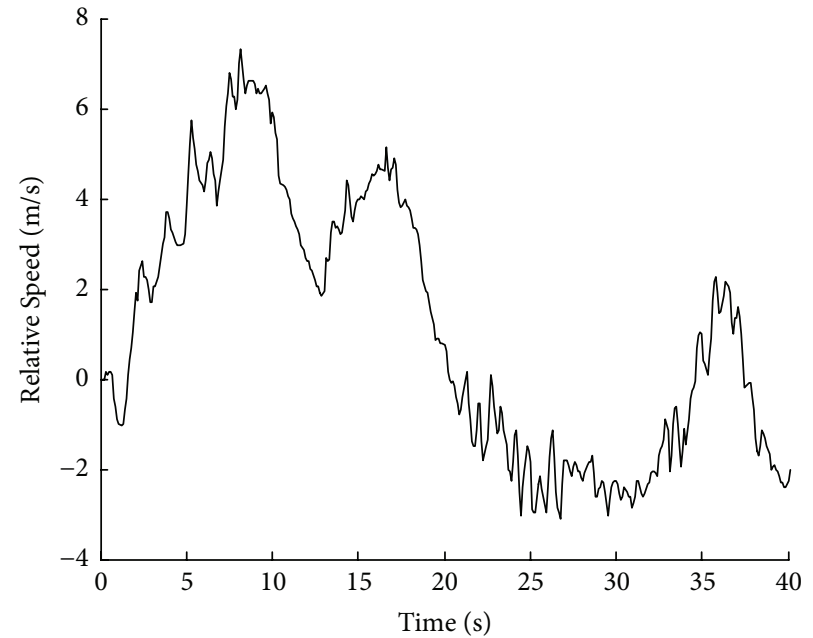

(b)

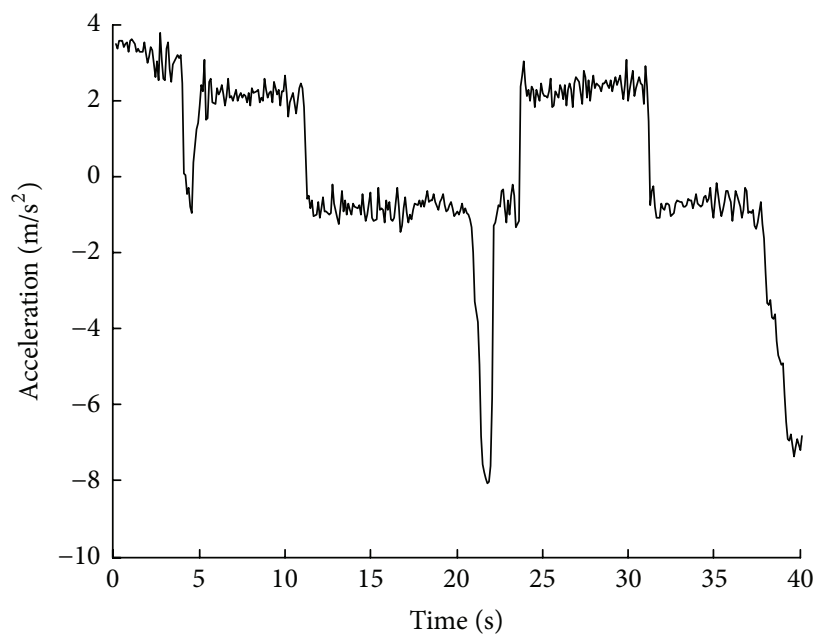

(c)

(d)

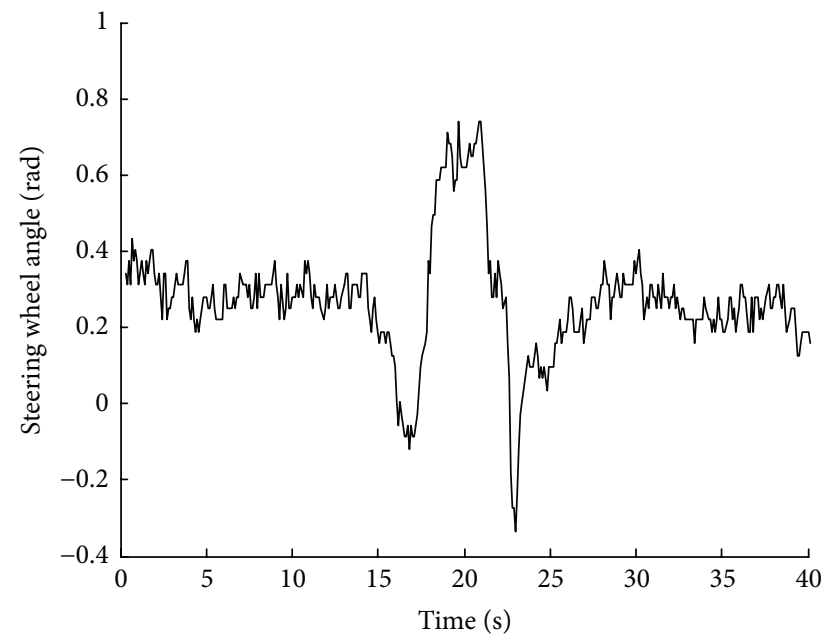

(e)

FIGURE 17: The information collected by sensors in experiment 2. (a) The distance between SV and LV. (b) The relative speed. (c) The vehicle speed and the single front wheel speed of SV. (d) The acceleration of SV. (e) The steering wheel angle of SV. 


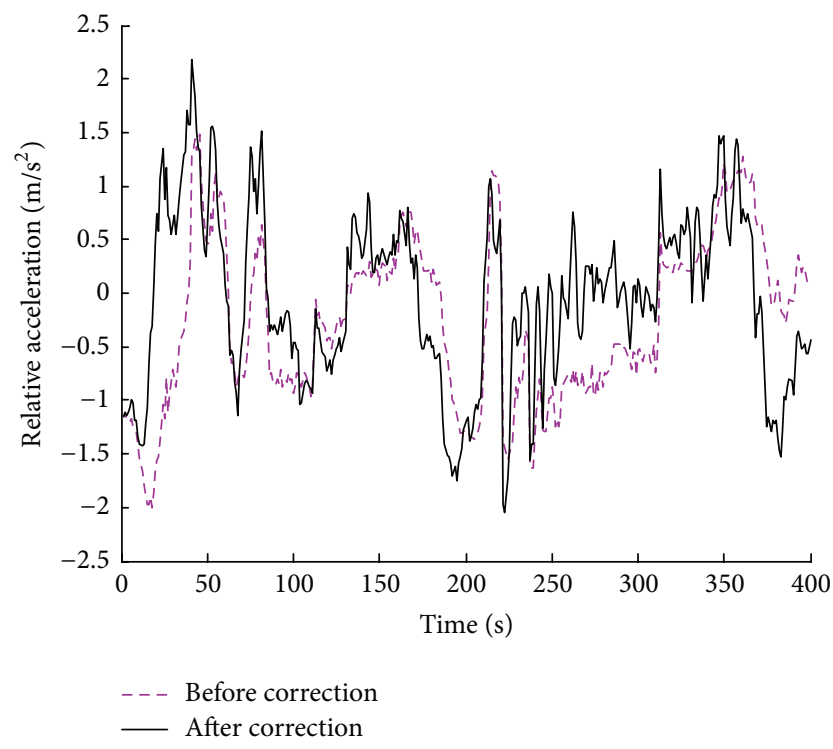

(a)

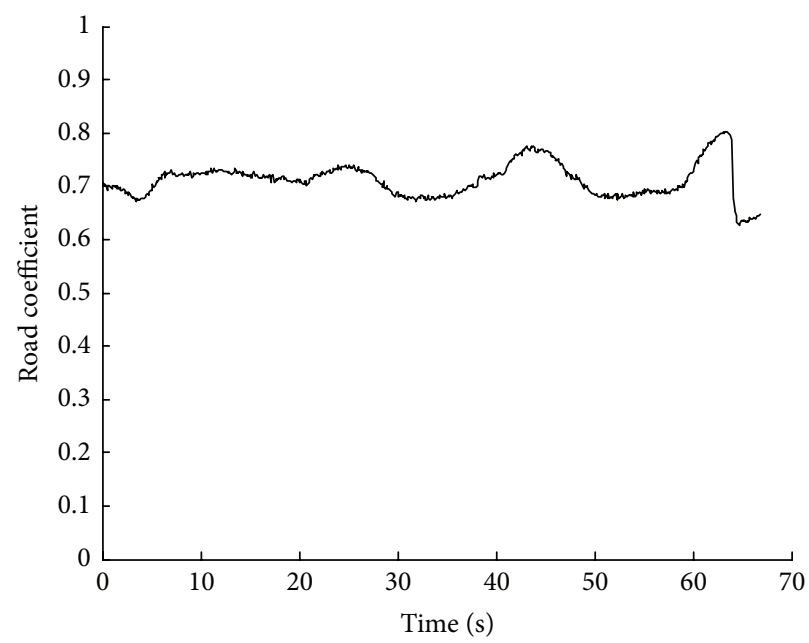

(c)

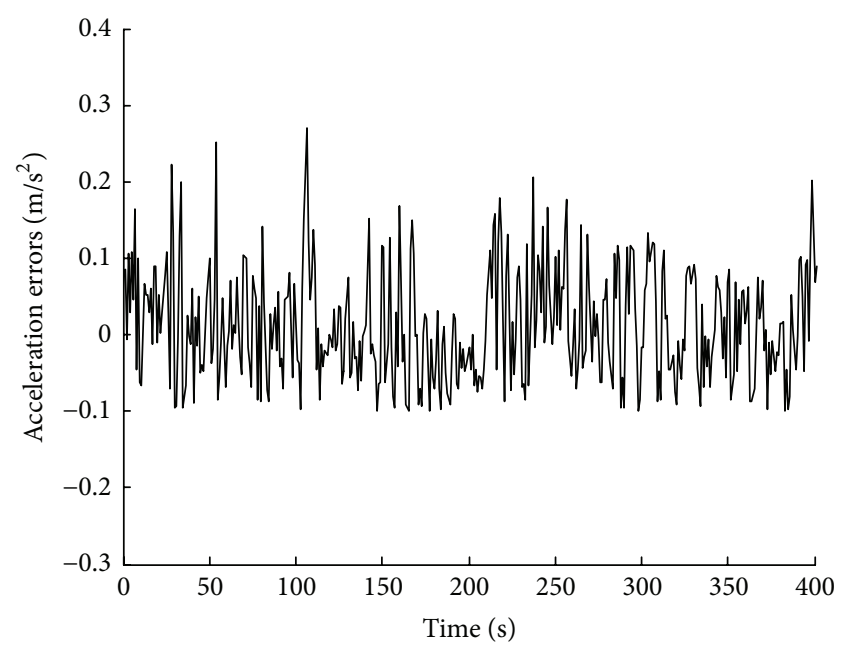

(b)

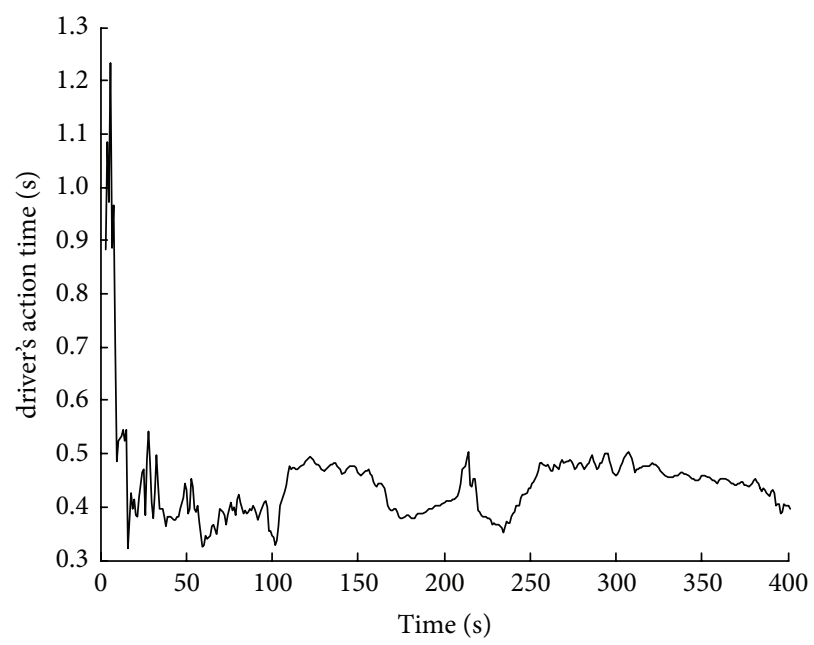

(d)

FIGURE 18: The key parameters estimation results of experiment 2. (a) The preliminary and final estimation results of relative acceleration. (b) The estimation error of relative acceleration. (c) The road friction coefficient. (d) The driver's reaction time.

coefficient is available. To illustrate the impact of the road friction coefficient on the safety distance, it is assumed that the road friction coefficient is 0.2 , and the responding safety distance is calculated (purple dashed line). Obviously, the calculated safety distance in the case that the road friction coefficient is 0.2 is far larger than that in the case that the road friction coefficient is 0.7 , and the warning is required at some time points. Thus we can see that the proposed method can adaptively adjust the safety distance according to the road condition. However, other methods cannot adapt to the road condition because the friction coefficient is assumed as a constant.

\section{Conclusions}

To realize reliable warning for rear-end collision of the vehicle, an adaptive distance-based warning strategy is proposed.
The proposed strategy is based on the parameter-adaptive and variable-threshold approaches.

In the proposed strategy, both the parameter estimation algorithms and the warning decision method have been developed to obtain higher performance. First, the estimation algorithms for some key parameters are developed, which can provide accurate information for the subsequent warning method. Further, the variable-threshold method has been designed to realize reliable warning decision. The effectiveness of the proposed strategy has been verified comprehensively. The simulation and experimental results show that the proposed strategy has higher adaptability and reliability than existing methods.

The key parameter estimation algorithms in proposed strategy can equally be applied to other vehicle active safety systems such as antiskid brake system (ABS) and traction control system (TCS). Our future work will concern how to 


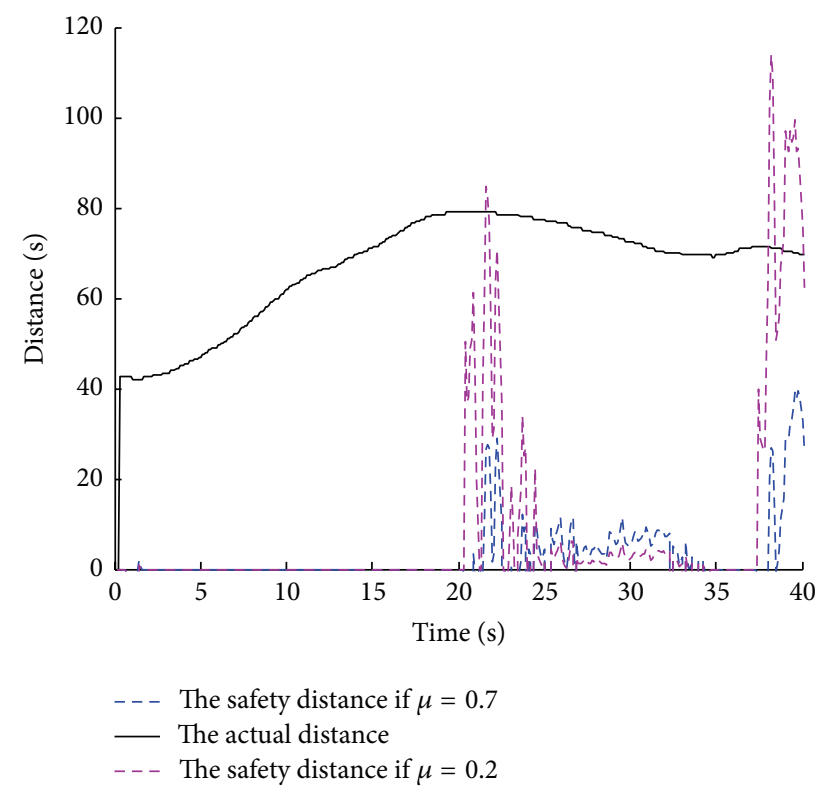

FIgURE 19: The safety distance of experiment 2.

further improve the performance of the proposed parameter estimation algorithms and to solve other issues involved in the study of rear-end collision warning system, such as the further elimination of false alarm and anticollision problems in curve road and multilane scene.

\section{Conflict of Interests}

The authors declare that there is no conflict of interests regarding the publication of this paper.

\section{Acknowledgments}

This work is supported by the National Natural Science Foundation of China (Grant no. 61273236), the Jiangsu Planned Projects for Postdoctoral Research Funds (Grant no. 1401012C), the Fundamental Research Funds for the Central Universities (Grant no. 2242015R20017), and the Project Funded by China Postdoctoral Science Foundation (Grant no. 2015M571631).

\section{References}

[1] N.-E. El Faouzi, H. Leung, and A. Kurian, "Data fusion in intelligent transportation systems: progress and challenges-a survey," Information Fusion, vol. 12, no. 1, pp. 4-10, 2011.

[2] M. Staubach, "Factors correlated with traffic accidents as a basis for evaluating Advanced Driver Assistance Systems," Accident Analysis and Prevention, vol. 41, no. 5, pp. 1025-1033, 2009.

[3] J. Santa, R. Toledo-Moreo, M. A. Zamora-Izquierdo, B. Úbeda, and A. F. Gómez-Skarmeta, "An analysis of communication and navigation issues in collision avoidance support systems," Transportation Research-Part C: Emerging Technologies, vol.18, no. 3, pp. 351-366, 2010.
[4] S. Shen, L. Hong, and S. Cong, "Reliable road vehicle collision prediction with constrained filtering," Signal Processing, vol. 86, no. 11, pp. 3339-3356, 2006.

[5] M. A. Hannan, A. Hussain, A. Mohamed, and S. A. Samad, "Development of an embedded vehicle safety system for frontal crash detection," International Journal of Crashworthiness, vol. 13, no. 5, pp. 579-587, 2008.

[6] Y.-S. Kim and K.-S. Hong, "An IMM algorithm for tracking maneuvering vehicles in an adaptive cruise control environment," International Journal of Control, Automation and Systems, vol. 2, no. 3, pp. 310-318, 2004.

[7] A. Polychronopoulos, M. Tsogas, A. J. Amditis, and L. Andreone, "Sensor fusion for predicting vehicles' path for collision avoidance systems," IEEE Transactions on Intelligent Transportation Systems, vol. 8, no. 3, pp. 549-562, 2007.

[8] J. Jansson and F. Gustafsson, "A framework and automotive application of collision avoidance decision making," Automatica, vol. 44, no. 9, pp. 2347-2351, 2008.

[9] S. Tabacu and N. Pandrea, "Numerical (analytical-based) model for the study of vehicle frontal collision," International Journal of Crashworthiness, vol. 13, no. 4, pp. 387-410, 2008.

[10] S. Moon, W. Cho, and K. Yi, "Intelligent vehicle safety control strategy in various driving situations," Vehicle System Dynamics, vol. 48, no. 1, pp. 537-554, 2010.

[11] A. H. Jamson, F. C. H. Lai, and O. M. J. Carsten, "Potential benefits of an adaptive forward collision warning system," Transportation Research Part C: Emerging Technologies, vol. 16, no. 4, pp. 471-484, 2008.

[12] A. Vahidi and A. Eskandarian, "Research advances in intelligent collision avoidance and adaptive cruise control," IEEE Transactions on Intelligent Transportation Systems, vol. 4, no. 3, pp. 132153, 2003.

[13] L. Cai, A. B. Rad, and W.-L. Chan, "An intelligent longitudinal controller for application in semiautonomous vehicles," IEEE Transactions on Industrial Electronics, vol. 57, no. 4, pp. 14871497, 2010.

[14] C. Garcia-Costa, E. Egea-Lopez, J. B. Tomas-Gabarron, J. Garcia-Haro, and Z. J. Haas, "A stochastic model for chain collisions of vehicles equipped with vehicular communications," IEEE Transactions on Intelligent Transportation Systems, vol. 13, no. 2, pp. 503-518, 2012.

[15] X. Zhao and Z. Gao, "A new car-following model: full velocity and acceleration difference model," The European Physical Journal B, vol. 47, no. 1, pp. 145-150, 2005.

[16] H.-H. Yang and H. Peng, "Development of an errorable carfollowing driver model," Vehicle System Dynamics, vol. 48, no. 6, pp. 751-773, 2010.

[17] Y. Xie, D. Lord, and Y. Zhang, "Predicting motor vehicle collisions using Bayesian neural network models: an empirical analysis," Accident Analysis and Prevention, vol. 39, no. 5, pp. 922-933, 2007.

[18] V. Milanés, J. Pérez, J. Godoy, and E. Onieva, "A fuzzy aid rearend collision warning/avoidance system," Expert Systems with Applications, vol. 39, no. 10, pp. 9097-9107, 2012.

[19] H.-H. Yang and H. Peng, "Development and evaluation of collision warning/collision avoidance algorithms using an errable driver model," Vehicle System Dynamics, vol. 48, no. 1, pp. 525$535,2010$.

[20] A. Tang and A. Yip, "Collision avoidance timing analysis of DSRC-based vehicles," Accident Analysis and Prevention, vol. 42, no. 1, pp. 182-195, 2010. 
[21] S. Park, J. Pil Hwang, E. Kim, and H.-J. Kang, "Vehicle tracking using a microwave radar for situation awareness," Control Engineering Practice, vol. 18, no. 4, pp. 383-395, 2010.

[22] A. Edelmayer, M. Miranda, and V. Nebehaj, "Cooperative federated filtering approach for enhanced position estimation and sensor fault tolerance in ad-hoc vehicle networks," IET Intelligent Transport Systems, vol. 4, no. 1, pp. 82-92, 2009.

[23] F. Gustafsson, F. Gunnarsson, N. Bergman et al., "Particle filters for positioning, navigation, and tracking," IEEE Transactions on Signal Processing, vol. 50, no. 2, pp. 425-437, 2002.

[24] R. Toledo-Moreo and M. A. Zamora-Izquierdo, "Collision avoidance support in roads with lateral and longitudinal maneuver prediction by fusing GPS/IMU and digital maps," Transportation Research Part C: Emerging Technologies, vol. 18, no. 4, pp. 611-625, 2010.

[25] H. Zhou, Tracking of maneuvering target [Ph.D. thesis], University of Minnesota, Minneapolis, Minn, USA, 1984.

[26] X. R. Li and V. P. Jilkov, "Survey of maneuvering target tracking. Part I: dynamic models," IEEE Transactions on Aerospace and Electronic Systems, vol. 39, no. 4, pp. 1333-1364, 2003.

[27] K. S. P. Kumar and H. Zhou, 'A 'current' statistical model and adaptive algorithm for estimating maneuvering targets," AlAA Journal of Guidance, Control, and Dynamics, vol. 7, no. 5, pp. 596-602, 1984.

[28] C. Lee, K. Hedrick, and K. Yi, "Real-time slip-based estimation of maximum tire-road friction coefficient," IEEE/ASME Transactions on Mechatronics, vol. 9, no. 2, pp. 454-458, 2004.

[29] M. Tanelli, L. Piroddi, and S. M. Savaresi, "Real-time identification of tire-road friction conditions," IET Control Theory and Applications, vol. 3, no. 7, pp. 891-906, 2009.

[30] C. Ahn, H. Peng, and H. E. Tseng, "Robust estimation of road friction coefficient using lateral and longitudinal vehicle dynamics," Vehicle System Dynamics, vol. 50, no. 6, pp. 961-985, 2012.

[31] R. Rajamani, D. N. Piyabongkarn, J. Y. Lew, K. Yi, and G. Phanomchoeng, "Tire-road friction-coefficient estimation," IEEE Control Systems Magazine, vol. 30, no. 4, pp. 54-69, 2010.

[32] F. Gustafsson, "Automotive safety systems, replacing costly sensors with software algorithms," IEEE Signal Processing Magazine, vol. 26, no. 4, pp. 32-47, 2009.

[33] M. Y. Bian, "Simplified tire model for longitudinal road friction estimation," Journal of Chongqing University of Technology: Natural Science, vol. 26, no. 1, pp. 1-5, 2012.

[34] J. Wang, L. Alexander, and R. Rajamani, "Friction estimation on highway vehicles using longitudinal measurements," Journal of Dynamic Systems, Measurement and Control, vol. 126, no. 2, pp. 265-275, 2004.

[35] S. Niu and W. Zhan, "Analysis of confidence lower limits of reliability and hazard rate for electronic stability control systems," Quality and Reliability Engineering International, vol. 29, no. 5, pp. 621-629, 2013.

[36] W. H. Wang, Y. Mao, J. Jin et al., “Driver's various information process and multi-ruled decision-making mechanism: a fundamental of intelligent driving shaping model," International Journal of Computational Intelligence Systems, vol. 4, no. 3, pp. 297-305, 2011.

[37] T. Wada, S. Doi, N. Tsuru, K. Isaji, and H. Kaneko, "Characterization of expert drivers' last-second braking and its application to a collision avoidance system," IEEE Transactions on Intelligent Transportation Systems, vol. 11, no. 2, pp. 413-422, 2010.
[38] G. P. Siegmund, D. J. King, and D. K. Mumford, "Correlation of steering behavior with heavy truck driver fatigue," $S A E$ Transactions, vol. 105, no. 6, pp. 1547-1568, 1996.

[39] L. M. Bergasa, J. Nuevo, M. A. Sotelo, R. Barea, and M. E. Lopez, "Real-time system for monitoring driver vigilance," IEEE Transactions on Intelligent Transportation Systems, vol. 7, no. 1, pp. 63-77, 2006.

[40] J. Peng, Y. Guo, R. Fu, W. Yuan, and C. Wang, "Multi-parameter prediction of drivers' lane-changing behaviour with neural network model," Applied Ergonomics, vol. 50, pp. 207-217, 2015.

[41] H. Jin and L. Li, "Dynamic network traffic flow prediction model based on modified quantum-behaved particle swarm optimization," Journal of Networks, vol. 8, no. 10, pp. 2332-2339, 2013. 


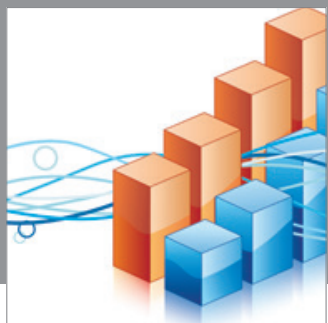

Advances in

Operations Research

mansans

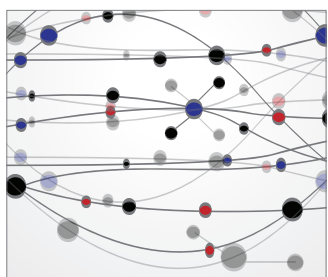

The Scientific World Journal
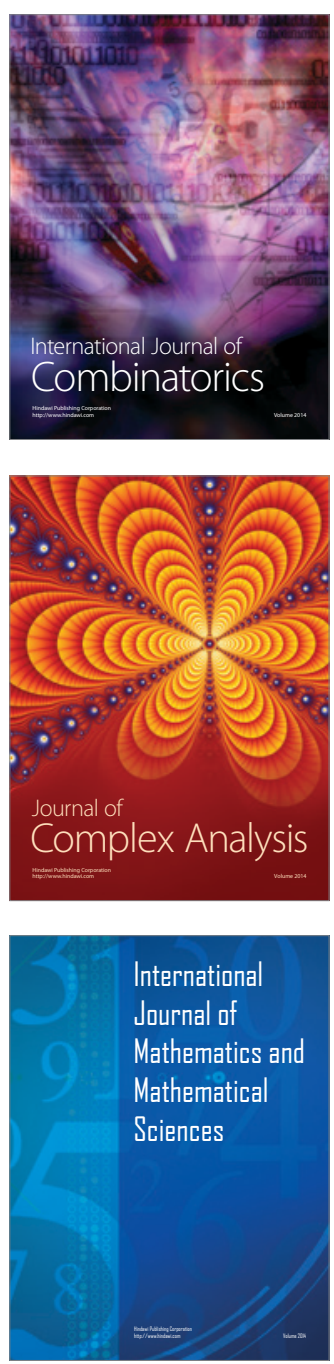
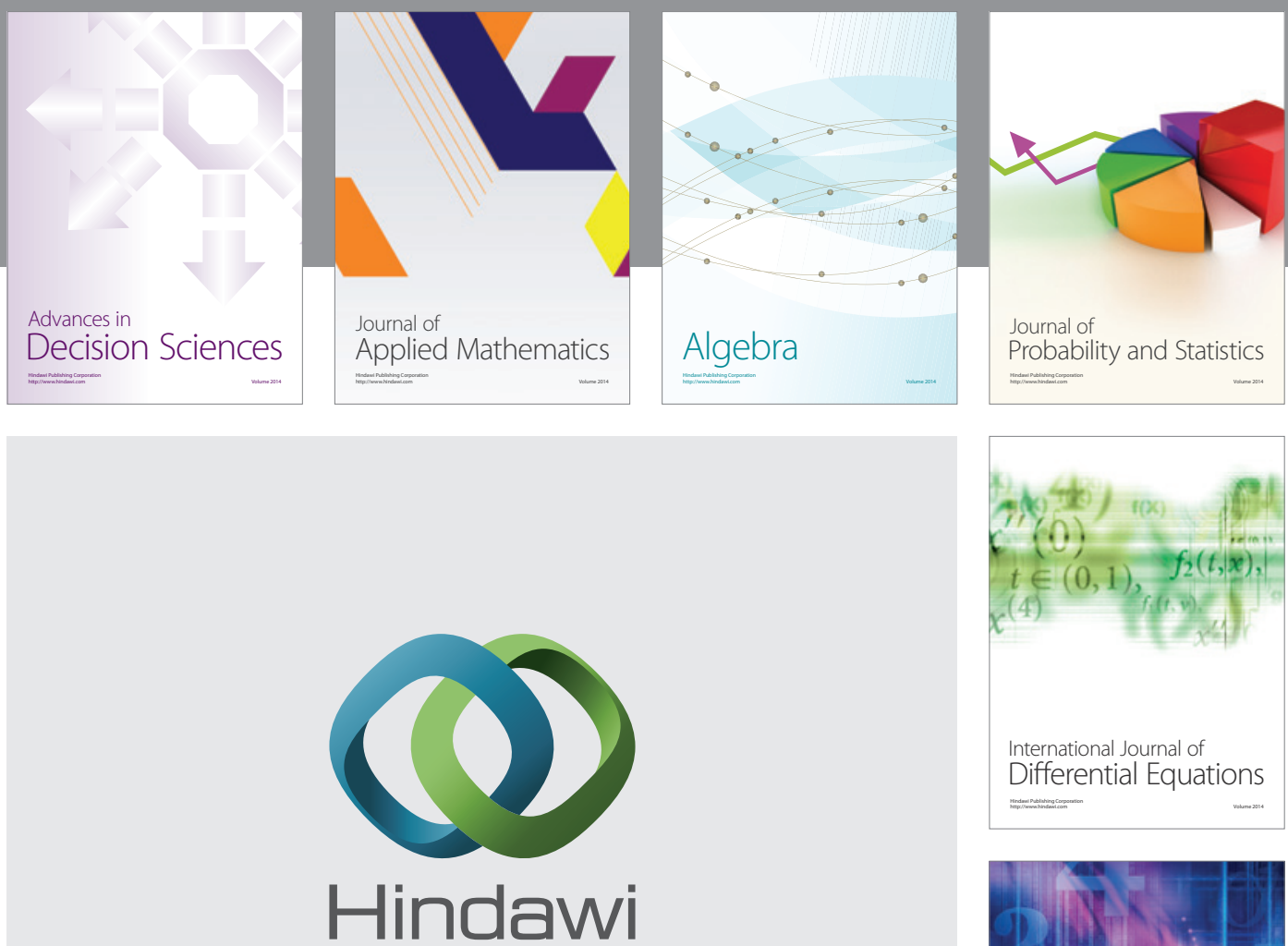

Submit your manuscripts at http://www.hindawi.com
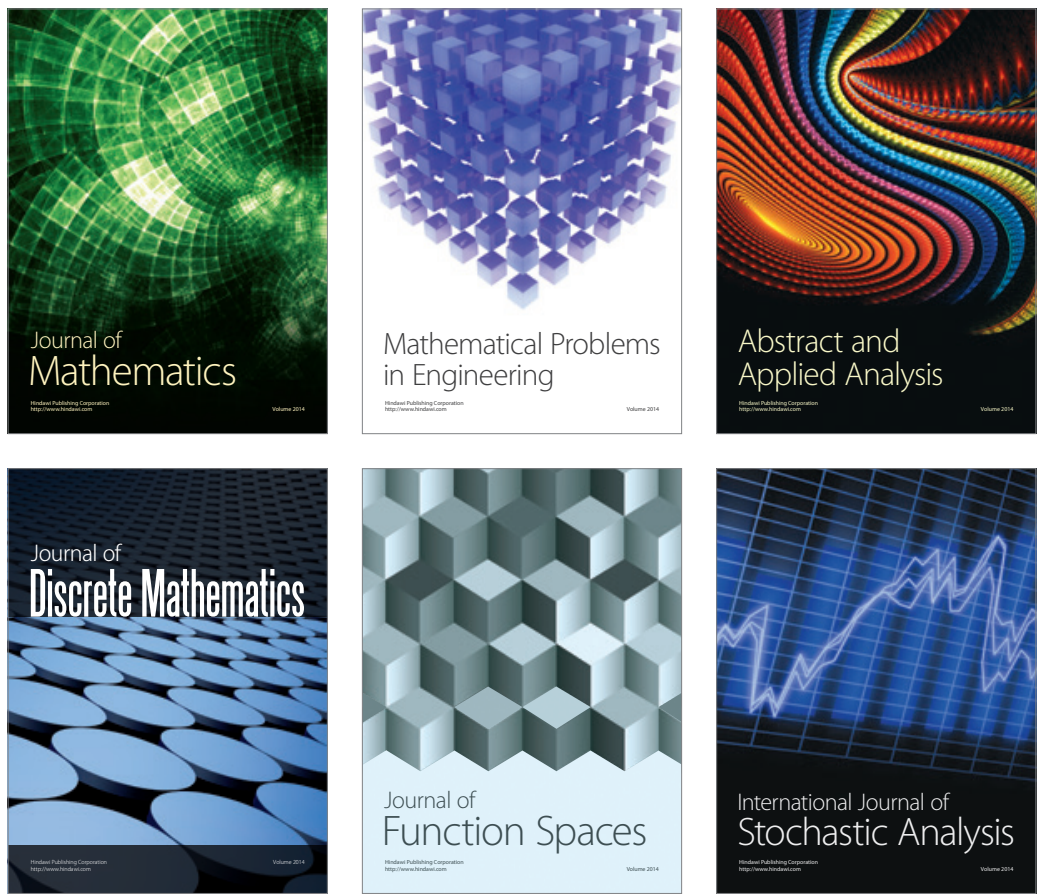

Journal of

Function Spaces

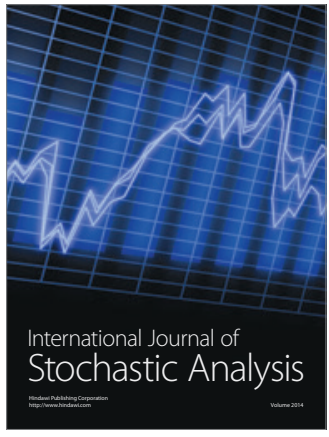

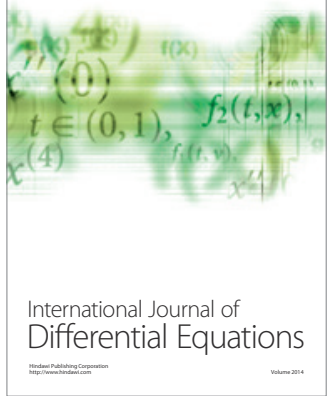
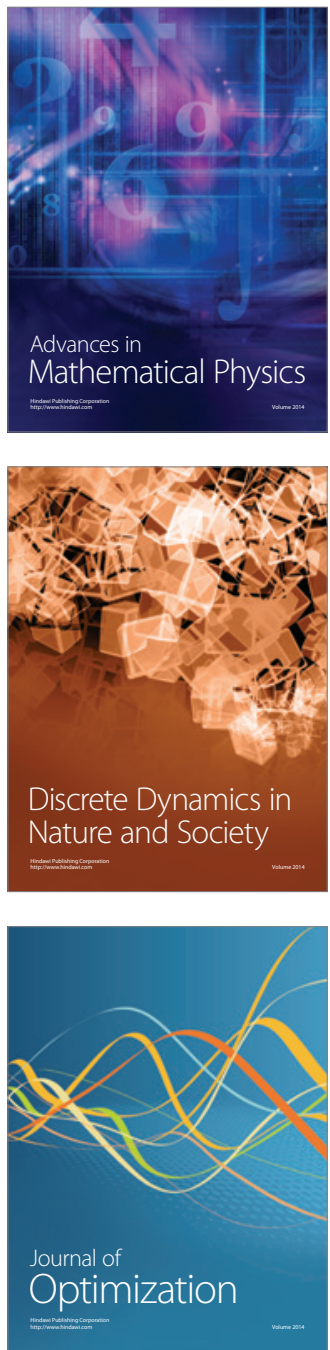